\title{
Measuring local moiré lattice heterogeneity of twisted bilayer graphene
}

\author{
Tjerk Benschop $\odot,{ }^{1, *}$ Tobias A. de Jong $\odot,{ }^{1, *}$ Petr Stepanov,,${ }^{2, *}$ Xiaobo Lu, ${ }^{2}$ Vincent Stalman, ${ }^{1}$ Sense Jan van der Molen $\odot,{ }^{1}$ \\ Dmitri K. Efetov (1), ${ }^{2}$ and Milan P. Allan ${ }^{1, \dagger}$ \\ ${ }^{1}$ Huygens-Kamerlingh Onnes Laboratory, Leiden Institute of Physics, Leiden University, Niels Bohrweg 2, 2333 CA Leiden, the Netherlands \\ ${ }^{2}$ ICFO - Institut de Ciencies Fotoniques, The Barcelona Institute of Science and Technology, 08860 Castelldefels, Barcelona, Spain
}

(Received 18 September 2020; revised 5 December 2020; accepted 15 January 2021; published 16 February 2021)

\begin{abstract}
We introduce a new method to continuously map inhomogeneities of a moiré lattice and apply it to large-area topographic images we measure on open-device twisted bilayer graphene (TBG). We show that the variation in the twist angle of a TBG device, which is frequently conjectured to be the reason for differences between devices with a supposed similar twist angle, is about $0.08^{\circ}$ around the average of $2.02^{\circ}$ over areas of several hundred nanometers, comparable to devices encapsulated between hexagonal boron nitride slabs. We distinguish between an effective twist angle and local anisotropy and relate the latter to heterostrain. Our results imply that for our devices, twist angle heterogeneity has an effect on the electronic structure roughly equal to that of local strain. The method introduced here is applicable to results from different imaging techniques and on different moiré materials.
\end{abstract}

DOI: 10.1103/PhysRevResearch.3.013153

\section{INTRODUCTION}

Stacking two sheets of identical periodic lattices with a small twist angle $\theta$ leads to a superperiodic lattice with moiré lattice constant $\lambda(\theta)$ much larger than the original lattice constant $a$ [Fig. 1(a)]. This new lattice is called a moiré lattice. When using atomic layers exfoliated from van der Waals materials and stacking them with a twist angle, the electronic and structural properties are modulated on the moiré length scale $\lambda(\theta)$, leading to the potential for new, emergent electronic properties of the moiré material [1,2].

Such new properties have been spectacularly demonstrated in twisted bilayer graphene (TBG) around the magic angle of $\theta \approx 1.1^{\circ}[3-11]$. In TBG, the moiré lattice modulates the interlayer coupling between the individual graphene sheets, as well as the van der Waals forces on the individual carbon atoms. The former leads to flat bands of low-kinetic-energy electrons [1]. The latter leads to a slight deformation of the graphene lattice and band gaps that separate the more localized electrons from the other bands [1]. When the flat bands are tuned to the Fermi level, they pair and condense into a superfluid at temperatures much higher than what one would naively expect at the low carrier densities observed in TBG [4]. Additionally, a variety of insulating and metallic behavior has been observed in TBG for different twist angles and band fillings $[3,5,6,12]$.

\footnotetext{
${ }^{*}$ These authors contributed equally to this work.

${ }^{\dagger}$ Corresponding author: milan.allan@gmail.com

Published by the American Physical Society under the terms of the Creative Commons Attribution 4.0 International license. Further distribution of this work must maintain attribution to the author(s) and the published article's title, journal citation, and DOI.
}

The kinetic energy of the electrons changes rapidly as the twist angle is varied, especially around the magic angle; therefore the fabrication of devices with just the right angle is key in making them superconducting. However, getting the right angle might not even be the most challenging aspect of fabricating high-quality superconducting TBG devices: Contaminations, internal stress, and heterogeneities of the twist angle are difficult to avoid. This is in part because the magic angle is not the lowest energy configuration and in part because of the strong forces associated with the tear-and-stack technique. Internal stress and heterogeneities are often conjectured to limit the quality of the devices and are attributed as the main causes for the variability between devices [13]. This holds especially for open devices that lack the hexagonal boron nitride (hBN) top layer; notably, such devices have never been found to superconduct. Measuring, visualizing, and characterizing heterogeneity in the twist angle and strain in TBG is thus crucial to understand and improve devices.

Probably the most complete visualization of inhomogeneity thus far has been obtained using scanning superconducting quantum interference device (SQUID)-on-tip microscopy (SOT) [14]. SOT measures the Landau levels as a function of location and thus has access to the local superlattice carrier density. On encapsulated devices, SOT has been used to visualize heterogeneity on length scales of a few micrometers with a resolution of several tens of nanometers, demonstrating that the twist angle varies by less than $4 \%$ [14]. While being a very precise measure of the local twist angle, SOT is also influenced by other factors, e.g., inhomogeneities of the chemical potential and the local magnetic screening. Other techniques to access homogeneity are nanospot angle-resolved photoemission spectroscopy (nano-ARPES) [15-17], which can image the full electronic structure in reciprocal space with a spatial resolution of circa $600 \mathrm{~nm}$, low-energy electron microscopy (LEEM) [15], which 


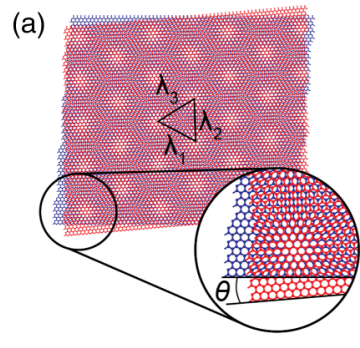

(b)

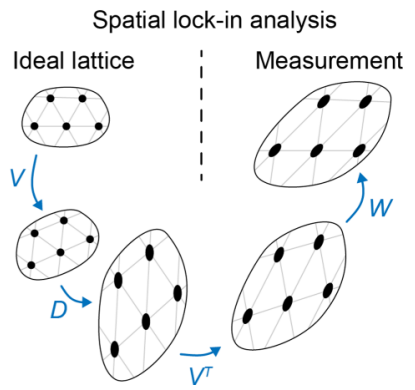

FIG. 1. (a) Moiré pattern created by stacking two hexagonal lattices with a twist angle of $5^{\circ}$. (b) Schematic representation of our spatial lock-in algorithm to map the local twist angle and anisotropy. The measured lattice can be thought of as the result of a series of transformations applied to an ideal lattice. The scaling transformation $D$ holds information about the local twist angle $\theta^{*}(\mathbf{r})$ and the intrinsic local strain present in the device, $\kappa(\mathbf{r})$. $V$ gives the direction of this local strain, $\psi(\mathbf{r})$. Finally, $W$ indicates the relative angle between the bilayer and the underlying $\mathrm{hBN}$ substrate.

can image structural inhomogeneities at twist angles lower and higher than the magic angle, conductive atomic-force microscopy (AFM) [18], nano-photocurrent mapping [19], which can measure the twist angle with a resolution on the order of $20 \mathrm{~nm}$, and scanning single-electron transistors [20], which can map the twist angle by measuring the inverse local compressibility. Finally, scanning tunneling microscopy (STM), the probe used in this study, has been used to measure both the topography and the local density of states of TBG, including the emergence of correlations at the magic angle [21-26].

In previous STM studies, two different methods have been used to determine the local twist angle. First, one can determine the twist angle using three neighboring moiré lattice sites in real space. The distances between each lattice site, $\lambda_{1}, \lambda_{2}$, and $\lambda_{3}$, are fit to a set of equations that yield the twist angle at a per-triangle resolution [Fig. 1(a)] [22] and, using a model with assumptions about the strain distribution in the two layers, an estimate for the heterostrain $\epsilon$.

A second method to determine the twist angle uses the Fourier transform of a real-space topography to determine the moiré wavelengths $\lambda_{j}$ in the three directions of the moiré lattice (in principle, two directions are fully determining the lattice, but often all three are used for a better signal-to-noise ratio). The twist angle is determined using $\lambda=\frac{a}{2 \sin \left(\frac{\theta}{2}\right)}$, where $\lambda=\frac{1}{3} \sum_{j=1}^{3} \lambda_{j}$ and $a$ is the lattice constant of graphene. Using the Fourier transform is generally more accurate than fitting three moiré lattice peaks, because it averages over the whole field of view, but this also limits its spatial resolution to the full field of view.

In this paper, we introduce an alternative method of quantifying and visualizing the heterogeneity in open devices, with sub-moiré lattice cell resolution over length scales of hundreds of nanometers. We develop a spatial lock-in method that enables one to map, with sub-moiré wavelength resolution, the local twist angle $\theta^{*}(\mathbf{r})$, the local moiré anisotropy $\kappa(\mathbf{r})$, and the anisotropy direction $\psi(\mathbf{r})$, as defined below. Notably, we can separate these effects from each other and from rotations of the lattice [Fig. 1(b)]. We then apply our method to determine the heterogeneity in open TBG devices.

\section{METHODS}

We fabricate our devices using the tear-and-stack method with a special focus on avoiding contamination to ensure the large clean areas needed for this study. A single graphene flake is precut into two halves with an AFM tip, ensuring initial crystallographic alignment between them. The first half is subsequently picked up with a hBN flake, mechanically exfoliated on $\mathrm{SiO}_{2} / \mathrm{Si}$ chip, and adhered to a polydimethylsiloxane (PDMS)/polycarbonate (PC) stamp at $\sim 100{ }^{\circ} \mathrm{C}$. The second half of the graphene flake is manually rotated to a target twist angle of $1.5^{\circ}-2.0^{\circ}$ and consequently picked up by the $\mathrm{hBN} /$ graphene stack on PC. In the next step, the PC layer is carefully peeled off of the initial PDMS stamp and placed on another PDMS stamp upside down. The sacrificial PC layer is then removed in 1-methyl-2-pyrrolidone. Subsequently, the $\mathrm{TBG} / \mathrm{hBN}$ heterostructure is transferred onto a target $\mathrm{SiO}_{2} / \mathrm{Si}$ substrate with a prepatterned navigation structure, two gold electrodes, and a graphite gate contacting one of them within the measurement area. We carefully align the $\mathrm{TBG} / \mathrm{hBN}$ stack with the local graphite gate to avoid short-circuiting. The second prepatterned gold electrode is used to electrically contact TBG using another graphite piece. The devices are inserted into our ultrahigh-vacuum setup and annealed at $350{ }^{\circ} \mathrm{C}$ for $12 \mathrm{~h}$ before inserting them into the low-temperature STM operating at $4.2 \mathrm{~K}$. The TBG samples are located using a capacitive navigation scheme [27].

\section{SPATIAL LOCK-IN ALGORITHM}

Figure 2(a) shows a topographic image where both the atomic lattice of the top graphene layer and the moiré lattice are resolved. The Fourier transform of the image shows the lattice peaks as well as the peaks from the moiré superlattice [Fig. 2(b), blue and green insets, respectively]. While such small fields of view are well suited for spectroscopy studies, we require large fields of view that encompass many moiré cells for the heterogeneity study using spatial lock-in detection presented here. Figure 2(c) shows an example.

The general method of spatial lock-in is illustrated in the left column of Fig. 3 for the one-dimensional case: The "measured" signal $S(x)$, a not-quite periodic signal, is multiplied with a reference signal, a perfectly periodic complex plane wave $S_{\text {ref }}(x)$. The phase of the resulting signal, when low-pass filtered, corresponds to the local phase of the original wave. To obtain the local variations in wavelength $\lambda(x)$ of the original wave, shown at the bottom left of Fig. 3, one calculates the derivative of the local phase. Spatial lock-in algorithms like this have been used previously in electron microscopy studies (known as geometric phase analysis) [28-30] and optical metrology [31]. In the context of STM, the most well-known application is known as the Lawler-Fujita algorithm [32]. Lawler, Fujita, and co-workers have, based on earlier work by Slezak et al. [33], introduced a lock-in algorithm to correct topographic images for drift by calculating the displacement field, i.e., the vectors that connect the coordinates of the measured images with the points of an ideal reference lattice. 
(a)

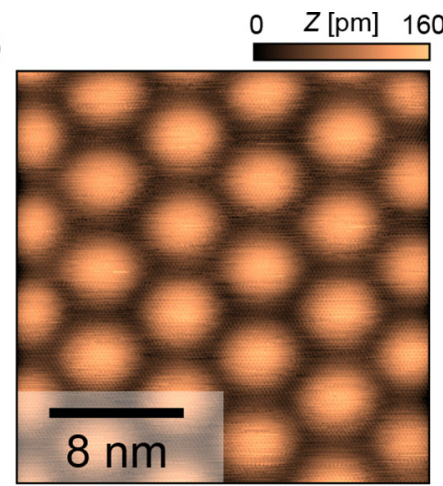

(b)

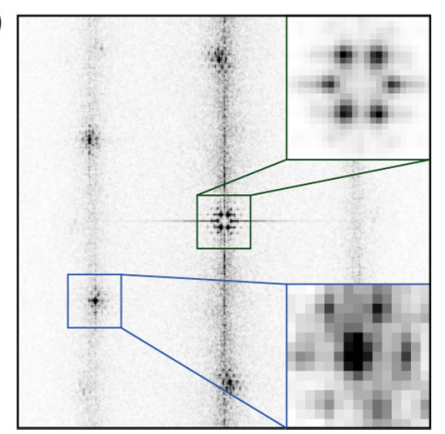

(c)

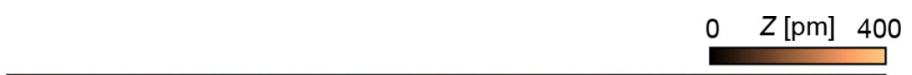

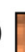

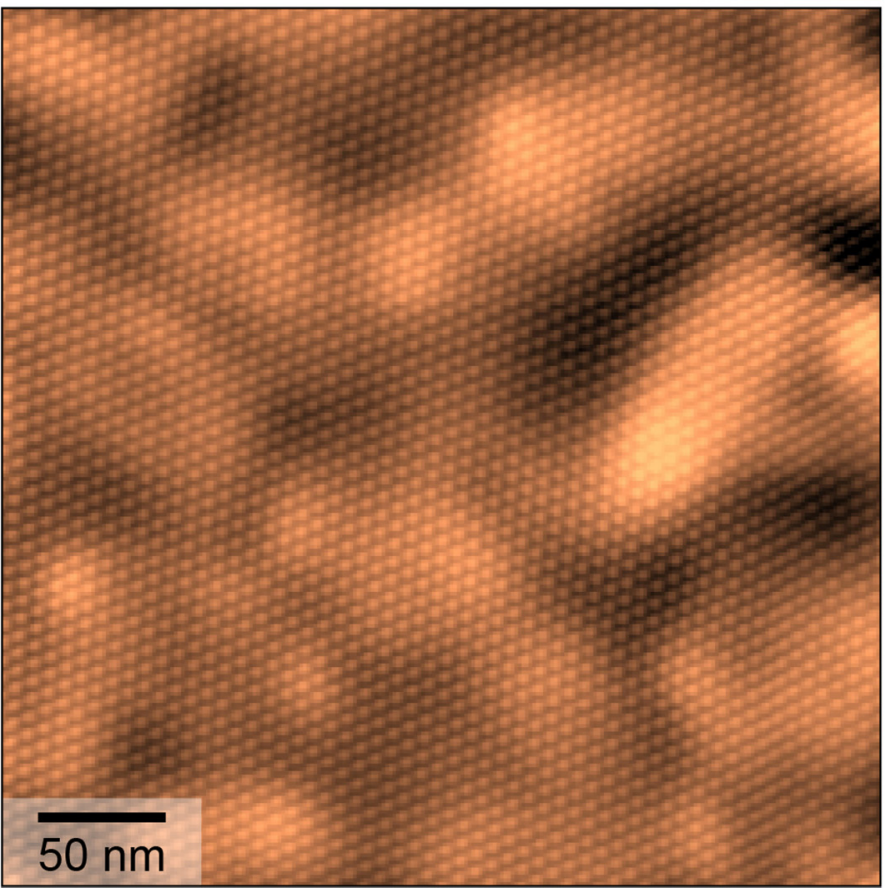

FIG. 2. (a) STM topography of a device with an average twist angle of $\theta=2.38^{\circ}$ (setup conditions: $V=250 \mathrm{mV}, I=100 \mathrm{pA}$ ). The topography shows both the atomic and moiré lattice. (b) Fourier transform of (a), with zoom-ins of the moiré peaks (green inset) and the bottom left atomic peak (blue inset). Satellite peaks of the moiré lattice are visible around the atomic peak as well. (c) Large-scale topography measured on a different device with an average twist angle of $2.02^{\circ}$ (setup conditions: $V=250 \mathrm{mV}, I=20 \mathrm{pA}$ ).

Our motivation here is different: We do not need to correct an imperfect image, but want to extract heterogeneities of the lattice.

To do so, we start with defining three reference plane waves $R_{j}(\mathbf{r})=e^{i \mathbf{q}_{j} \cdot \mathbf{r}}, j \in\{1,2,3\}$, where the reference wave vectors $q_{j}$ are determined by simultaneously fitting six Gaussians to the Bragg peaks in the Fourier transform of the topography [Fig. 4(b)]. In order to measure deviations from an isotropic triangular lattice, we force the reference wave vectors to be of
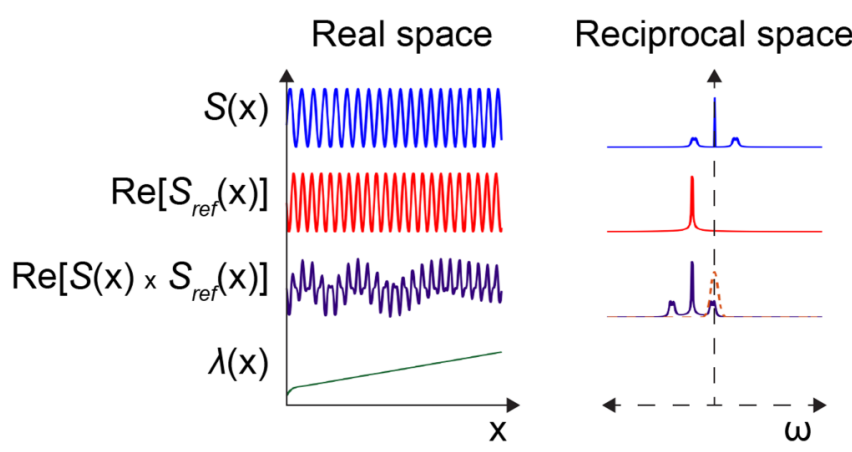

FIG. 3. Lock-in in 1D. The panels in the left column show, from top to bottom, the signal (an almost periodic sinusoid), the real part of the reference, the real part of the product of the signal and reference, and the wavelength calculated by taking the gradient of the phase of the product signal. The right column displays the Fourier transform of the (complex) signals in the left column. Finally, in the bottom right curve, the orange dashed line represents the Gaussian filter used for the lock-in procedure. equal magnitude and $60^{\circ}$ with respect to each other (although see Appendix A4 on the choice of reference vectors). The reference lattice is then defined as the real part of the sum of the reference plane waves, i.e., $T_{r}(\mathbf{r})=\operatorname{Re}\left[T_{0} \sum_{j} R_{j}(\mathbf{r})\right]=$ $T_{0} \sum_{j} \cos \left(\mathbf{q}_{j} \cdot \mathbf{r}\right)$, where $T_{0}$ is the average amplitude.

The transformation between the measured lattice, $T_{m}(\mathbf{r})$, and this perfectly periodic, hexagonal reference lattice, $T_{r}(\mathbf{r})$, can approximately be parametrized as the shifts between points in the moiré lattice and corresponding points in the reference lattice. To this end, we introduce the displacement field $\mathbf{u}(\mathbf{r})$ in the following manner:

$$
T_{m}(\mathbf{r})=T_{r}[\mathbf{r}+\mathbf{u}(\mathbf{r})]=T_{0} \sum_{j} \cos \left\{\mathbf{q}_{j} \cdot[\mathbf{r}+\mathbf{u}(\mathbf{r})]\right\} .
$$

To extract the displacement field from our data, each reference signal is multiplied with the original topographic image and low-pass filtered with a Gaussian window. This operation corresponds to convolution of the original topographic image with the plane wave encompassed by a Gaussian, calculating the relevant wave vector component of the ultimately small window two-dimensional (2D) Fourier transform. The window of the Gaussian filter needs to be chosen large enough (small enough in frequency space) in order to exclude larger frequencies but simultaneously small enough (big enough in frequency space) to maintain good spatial resolution (Appendix A). A lower limit on the filter size is put in place by higher frequencies: If the filter chosen is too small (too large in frequency space), more of the higher frequencies become included in the filter window reducing the signal-to-noise ratio. 
(a)

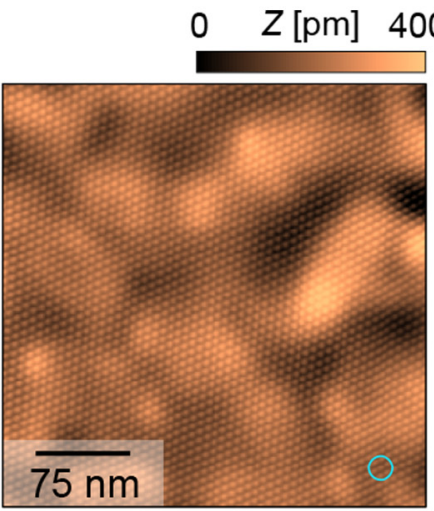

(d)
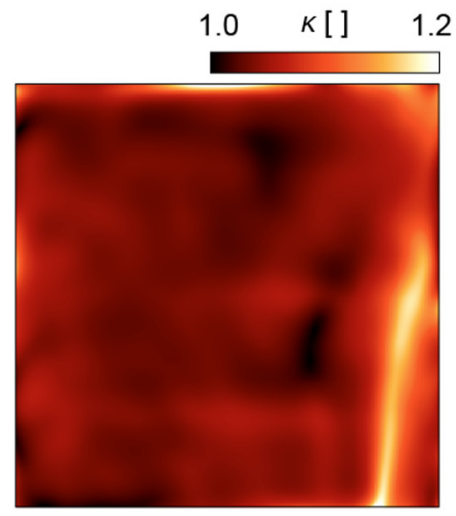

(b)

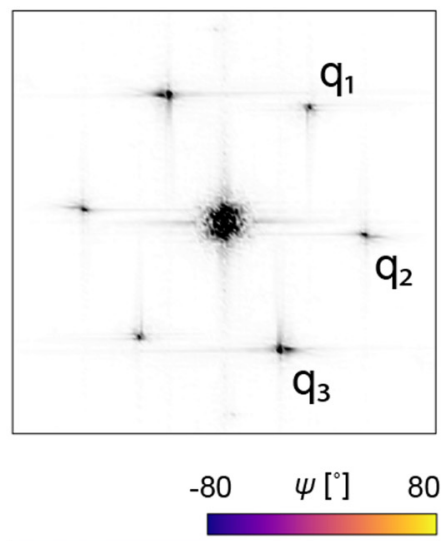

(e)

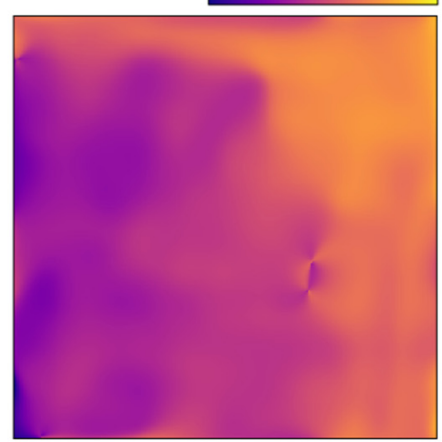

(c)
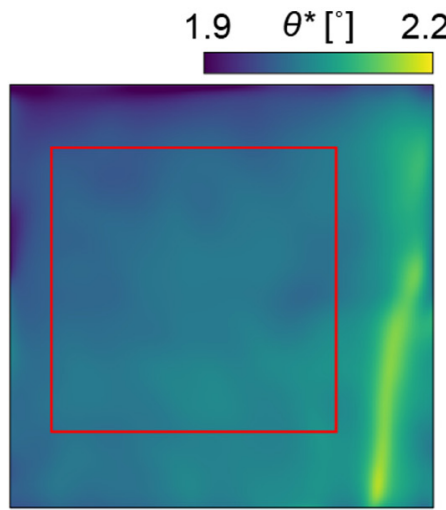

(f)
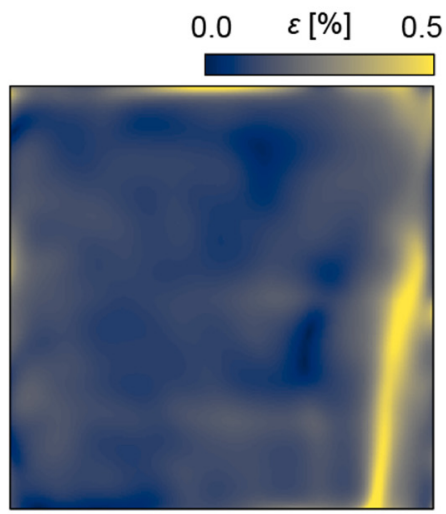

FIG. 4. (a) STM topography of a device with an average twist angle of $2.02^{\circ}[V=250 \mathrm{mV}, I=20 \mathrm{pA}$, same data as in Fig. 2(c)]. The blue circle at the bottom right indicates the size of the filter used by the algorithm (see text). (b) Fourier transform of (a), showing the Bragg peaks of the moiré lattice visible in the image. The Bragg peaks are labeled $q_{1}-q_{3}$. (c) Effective twist angle map extracted from (a), by the algorithm discussed. The red square indicates the area over which the average twist angle and standard deviation are calculated. (d) Local moiré anisotropy map $\kappa(\mathbf{r})$ extracted by the algorithm from (a). (e) Local moiré anisotropy direction $\psi(\mathbf{r})$ extracted by the algorithm from (a). (f) Heterostrain map extracted as described in the text.

In practice, a filter width of a few periods is used, as illustrated by the circle in Fig. 4(a). The local phase of the result of this operation corresponds to the local shift between the real image and the reference wave, or more precisely $\phi_{j}(\mathbf{r})=\mathbf{q}_{j} \cdot \mathbf{u}(\mathbf{r})$ (Appendix A).

This local phase is $2 \pi$ periodic and needs to be phase unwrapped to remove discontinuities. After phase unwrapping, the displacement field $\mathbf{u}(\mathbf{r})$ can be extracted from two of the phase maps by pixelwise multiplication with $Q^{-1}$, the inverse of a matrix containing the wave vectors used (although not applied here, using all three wave vectors is more involved but can be beneficial for situations with low signal-to-noise ratio, as detailed in Appendix A4).

In a second step, we decompose the obtained displacement field $\mathbf{u}(\mathbf{r})$ into the local effective twist angle $\theta^{*}(\mathbf{r})$ and the local moiré anisotropy magnitude and direction, $\kappa(\mathbf{r})$ and $\psi(\mathbf{r})$, respectively. To that end, we consider the Jacobian of the transformation, $J=I+\nabla \mathbf{u}$, which is the displacement gradient tensor that describes the transformation of an infinitesimal triangle at each position. The polar decomposition $J=W A$ splits $J$ into the product of the unitary matrix $W$, describing the local rotation of the lattice, and a matrix $A$, describing the local scaling and anisotropy. This matrix $A$ can be further decomposed into a (unitary) rotation matrix $V$, indicating the major and minor axes of scaling and a diagonal scaling matrix $D$ such that $J=W A=W V^{\top} D V$. This final decomposition is illustrated in Fig. 1(b) and makes it straightforward to extract relevant quantities. The change in density of unit cells is equal to the change in area under the effect of the deformation gradient tensor; hence the geometric mean of the scaling elements in the diagonal of $D, \sqrt{d_{1} d_{2}}=\sqrt{\operatorname{det}(J)}$, allows us to calculate the wavelength of the moiré lattice and, consequently, the local twist angle (Appendix A). Furthermore, the local anisotropy magnitude $\kappa(\mathbf{r})$ is calculated by taking the ratio of the scaling elements that make up $D, \kappa=\frac{d_{1}}{d_{2}}$, where $d_{1}>d_{2}$. Defined in this way, $\kappa=1$ indicates an isotropic lattice, and $\kappa>1$ indicates an anisotropy of the moiré lattice in the direction given by $\psi$, the angle corresponding to the rotation corresponding to $V$. Lastly, the rotation of the total lattice, corresponding to $W$, is left unattended, as a rotation of the full lattice should not directly influence the physics at play, although we point out that it does describe the variations of the rotation with respect to the $\mathrm{hBN}$ substrate.

\section{RESULTS}

Figure 4(c) shows the effective twist angle $\theta^{*}(\mathbf{r})$, Fig. 4(d) shows the local anisotropy $\kappa(\mathbf{r})$, and Fig. 4(e) shows the angle of major scaling $\psi(\mathbf{r})$, all as a function of location for open-device TBG. The maps show rather smooth variations with an exception in the bottom right corner of the field of view, where an apparent vertical feature appears. This feature 
is only barely visible in the topography itself, showcasing the sensitivity of our method. The origin of this particular vertical stripe remains unclear, and no such peculiarities were observed in our other data (Appendix G).

The overall twist angle heterogeneity in the image in Fig. 4(c), excluding border effects, is $0.033^{\circ}$ (standard deviation) or $0.23^{\circ}$ peak to peak. We find areas of hundreds of nanometers with a standard deviation of the twist angle of $0.02^{\circ}$ and a peak-to-peak variation of $0.08^{\circ}$, e.g., in the area marked by a red square in Fig. 4(c). A good estimate of the accuracy of our method can be made by applying the conventional Lawler-Fujita algorithm [32] and using spatial lock-in to extract the residual displacement field (Appendix E). We find residual twist angle variations more than one order of magnitude smaller than the originally found values, underlining the accuracy of our method. We further note that this is achieved with a pixel density corresponding to $\sim 5$ pixels per moiré lattice constant, which makes implementation of the conventional heterostrain model challenging (Appendix J).

Our result allows for a first comparison between open and encapsulated devices. For the latter, we compare our results with results from SOT [14]. SOT measures the superlattice density $n_{s}(\mathbf{r})$, which scales directly with the size of the unit cell. We note that SOT does not differentiate between heterogeneity of the chemical potential, strain, and twist angle, which can all influence $n_{s}(\mathbf{r})$.

To make a comparison between SOT and our data, one has to take into account the difference in the width of the point spread function (PSF). As this width is around $30 \mathrm{~nm}$ for SOT, we artificially broaden the PSF of our data to match (Appendix I), which naturally leads to a reduction of both the peak-to-peak spread and the standard deviation. In the full field of view, including the bright vertical feature, we find a peak-to-peak spread of $0.20^{\circ}$ and a standard deviation of $0.036^{\circ}$. These numbers are similar among different devices of similar twist angle (Appendix K) and measured for areas of several hundreds of nanometers across.

Interestingly, this result matches rather well with the result from SOT on encapsulated devices, despite the lack of a stabilizing top hBN slab in our devices. This implies that open devices can rival the quality of encapsulated devices, at least in terms of twist angle homogeneity.

Our results then raise the following question: Why have open devices never been shown to superconduct or to show spectral gaps in low-temperature tunneling experiments? Assuming that the mechanical properties of the bilayer do not change drastically as the angle of reconstruction is approached $\left(\theta \approx 1.0^{\circ}\right)$, our experiments suggest that the homogeneity of the TBG itself cannot be the only reason. Instead, another reason might be the absence of a second hBN layer encapsulating the bilayer, despite $\mathrm{hBN}$ often being neglected in theoretical studies due to its supposed weak interaction. Furthermore, the second hBN layer creates a near-symmetric environment for the bilayer. We speculate that the breaking of this symmetry may be the basis for the lack of superconductivity in open devices. However, more careful transport investigations of open devices are necessary to confirm this hypothesis.

The local anisotropy parameter $\kappa(\mathbf{r})$ discussed here can be related to heterostrain, following the model of Kerelsky et al.
[22]. Here, it is assumed that one of the graphene sheets is strained with a uniaxial strain $\epsilon(\mathbf{r})$, while the other one is unaffected and only undergoes a rotation. To connect to our measurements, we note that for small average twist angles, the displacement field of the moiré lattice is related to relative displacement of the constituting layers by the following formula: $(\langle J\rangle-I) \cdot \mathbf{u}_{\text {moiré }}(\mathbf{r})=\mathbf{u}_{\downarrow}(\mathbf{r})-\mathbf{u}_{\uparrow}(\mathbf{r})=\mathbf{u}_{\sim}(\mathbf{r})$, where $\langle J\rangle$ is the Jacobian corresponding to the average angle between the layers and $\mathbf{u} \sim(\mathbf{r})$ is the relative displacement field experienced between the two sheets (Appendix B). The relative displacement field can be decomposed in the same way as before, where the angle corresponding to $W$ now corresponds to the deviation of the twist angle between the two sheets from the average twist angle, and the local anisotropy $\kappa(\mathbf{r})$ and $\psi(\mathbf{r})$ obtained from the resulting scaling matrix indicate the relative strain between the layers. Furthermore, from the resulting scaling matrix elements, we can calculate the magnitude of the strain applied to the deformed sheet, $\epsilon(\mathbf{r})$ (Appendix B). We show the resulting $\epsilon(\mathbf{r})$ in Fig. 4(f). On average, we find that $\epsilon=0.14 \%$ with a standard deviation of $0.09 \%$.

It is interesting to compare the numbers for strain and twist angle heterogeneity, and their respective influence on the electronic structure of TBG. Calculations using a continuum model have considered both strain and twist angle changes in TBG samples close to the magic angle [34]. It was shown that a heterostrain of $\epsilon \approx 0.1 \%$ results in a splitting of the van Hove singularities of approximately $5 \mathrm{meV}$. This is comparable to variations in the twist angle of about $0.03^{\circ}$, which we obtain by interpolating the relation between twist angle and van Hove splitting given in Ref. [35]. Furthermore, stress can cause strong qualitative changes to the electronic structure including new van Hove singularities for $\epsilon \approx 0.5 \%$. If we compare these numbers with our measurements, we conclude that there is a roughly equal effect of the observed strain and twist angle inhomogeneity, suggesting that both have to be taken into account when fabricating samples, as both effects significantly alter the electronic structure compared with a perfect lattice.

Before concluding, we want to address one potential challenge of the method introduced here: It is also sensitive to piezo drift. Piezo drift occurs in STM experiments due to thermal fluctuations that influence the piezo, due to piezo relaxation after a change of field of view or due to the piezo relaxation from the movement necessary to take the topography. The former two effects change over time. The latter effect depends on the speed with which the topography is measured. To check the validity of this procedure, we have repeated the above procedures for different topographies in the same field of view, taken with different scan speeds at different times. As we show in detail in Appendix F, these different measurements yield very similar results, demonstrating that the twist angle variations we measure are intrinsic and not a consequence of piezoelectric drift.

\section{CONCLUSION}

In this paper, we have visualized and characterized structural heterogeneity in TBG, demonstrating peak-to-peak variations in the twist angle of roughly $0.08^{\circ}$ over areas of hundreds of nanometers. While our samples exhibit an 
average twist angle higher than the magic angle, we expect the issues to be similar as long as the twist angle is above the reconstruction that occurs for twist angles $\lesssim 1^{\circ}$. This indicates that the best open-device TBG could, purely based on homogeneity of the twist angle, superconduct and that lack of experimental evidence thereof suggests a critical role of the missing hBN top layer. The spatial lock-in algorithm we introduced is in principle applicable to a variety of different moiré materials and, additionally, may also be usable in a different context, e.g., in determining the topological properties of band structures through quasiparticle interference (QPI) measurements [36]. We anticipate that this algorithm can be applied to other microscopy probes as well, including AFM and LEEM. Lastly, by presenting our results in the way we did, we hope to pave the way for further studies, especially for correlating electronic and spatial properties by combining with theoretical models such as the ones presented in Refs. [34,37,38].

\section{ACKNOWLEDGMENTS}

We thank H. Zandvliet, P. Koenraad, P. Neu, and R. Wijgman for valuable discussions. Furthermore, we thank K. van Oosten, F. Groenewoud, D. Scholma, and T. Mechielsen for technical support. This work was supported by the European Research Council (ERC StG SpinMelt, 758572) and by the Dutch Research Council (NWO), as part of the Frontiers of Nanoscience program, as well as through a Vidi grant (Vidi talent scheme, Project No. 680-47-536). D.K.E. acknowledges support from the Ministry of Economy and Competitiveness of Spain through the "Severo Ochoa" program for Centres of Excellence in R\&D (Grant No. SE5-0522), Fundació Privada Cellex, Fundació Privada Mir-Puig, the Generalitat de Catalunya through the CERCA program, the Horizon 2020 program under Grant Agreement No. 820378 (project 2D-SIPC), and the La Caixa Foundation.

\section{APPENDIX A: SPATIAL LOCK-IN ALGORITHM}

\section{Deformations of a lattice}

We perform lock-in measurements on images that clearly display a periodic lattice. In STM, this implies that we can use any topography of sufficient quality that displays the crystal lattice. The idea is to use a lock-in measurement in order to find a transformation of coordinates between the measured, "distorted" image and its pristine, undeformed equivalent (in this paper, a perfect triangular lattice). Defining the measured and pristine images as $T_{m}(\mathbf{r})$ and $T_{r}\left(\mathbf{r}^{\prime}\right)$, respectively, both with measurement coordinates $\mathbf{r}=(x, y) \in \mathbb{R}^{2}$ and lattice coordinates $\mathbf{r}^{\prime}=\left(x^{\prime}, y^{\prime}\right) \in \mathbb{R}^{2}$, the following relation holds:

$$
T_{m}(\mathbf{r})=T_{r}[\mathbf{r}+\mathbf{u}(\mathbf{r})]=T_{r}[\mathbf{f}(\mathbf{r})]=T_{r}\left(\mathbf{r}^{\prime}\right)=T_{m}\left[\mathbf{f}^{-1}\left(\mathbf{r}^{\prime}\right)\right],
$$

where the transformation from measurement coordinates to lattice coordinates is given by

$$
\mathbf{f}(\mathbf{r})=\mathbf{r}+\mathbf{u}(\mathbf{r})=\mathbf{r}^{\prime} .
$$

Here, $\mathbf{u}(\mathbf{r})$ is called the displacement field, connecting the measurement coordinates to the lattice coordinates, as is well established in continuum mechanics. For convenience, we also define the inverse displacement

$$
\mathbf{u}^{\prime}\left(\mathbf{r}^{\prime}\right):=\mathbf{f}^{-1}\left(\mathbf{r}^{\prime}\right)-\mathbf{r}^{\prime}=\mathbf{r}-\mathbf{r}^{\prime} .
$$

Note that by substitution, we have the following relation between forward and inverse displacement:

$$
\mathbf{u}^{\prime}\left(\mathbf{r}^{\prime}\right)=\mathbf{f}^{-1}[\mathbf{f}(\mathbf{r})]-[\mathbf{r}+\mathbf{u}(\mathbf{r})]=-\mathbf{u}(\mathbf{r}) .
$$

With this, we can express the pristine image at lattice coordinates in terms of the measured image:

$$
\begin{aligned}
T_{r}\left(\mathbf{r}^{\prime}\right) & =T_{m}\left[\mathbf{f}^{-1}\left(\mathbf{r}^{\prime}\right)\right]=T_{m}\left[\mathbf{r}^{\prime}+\mathbf{u}^{\prime}\left(\mathbf{r}^{\prime}\right)\right] \\
& =T_{m}\left[\mathbf{r}^{\prime}-\mathbf{u}(\mathbf{r})\right] \\
& =T_{m}\left\{\mathbf{r}^{\prime}-\mathbf{u}\left[\mathbf{r}^{\prime}-\mathbf{u}(\mathbf{r})\right]\right\} \\
& \approx T_{m}\left\{\mathbf{r}^{\prime}-\left[\mathbf{u}\left(\mathbf{r}^{\prime}\right)-(\nabla \mathbf{u})\left(\mathbf{r}^{\prime}-\mathbf{r}\right)\right]\right\} \\
& =T_{m}\left\{\mathbf{r}^{\prime}-\left[\mathbf{u}\left(\mathbf{r}^{\prime}\right)-(\nabla \mathbf{u}) \mathbf{u}(\mathbf{r})\right]\right\} \\
& =T_{m}\left\{\mathbf{r}^{\prime}-\mathbf{u}\left(\mathbf{r}^{\prime}\right)-(\nabla \mathbf{u}) \mathbf{u}^{\prime}\left(\mathbf{r}^{\prime}\right)\right\} .
\end{aligned}
$$

Therefore, if we can determine $\mathbf{u}(\mathbf{r})$, and thereby $\mathbf{u}^{\prime}\left(\mathbf{r}^{\prime}\right)$, we can reconstruct the pristine image. This is the idea of the Lawler-Fujita reconstruction algorithm [32]. In their original paper, Lawler, Fujita, and co-workers [32] use $\mathbf{u}^{\prime}\left(\mathbf{r}^{\prime}\right)=$ $-\mathbf{u}\left(\mathbf{r}^{\prime}\right)$, which is a good approximation if $\mathbf{u}$ varies slowly.

\section{Properties of the deformation}

The displacement field $\mathbf{u}(\mathbf{r})$, as defined above, fully describes the deformation of the lattice but does not directly provide insight into the relevant properties. To that end, we first define the Jacobian of the transformation $\mathbf{f}$,

$$
J \equiv \nabla \mathbf{f}=\mathbb{1}+\nabla \mathbf{u}
$$

where $\nabla \mathbf{u}$ is the Jacobian of the displacement field. In continuum mechanics terminology, $\nabla \mathbf{u}$ is the deformation gradient tensor, and in canonical terms it is defined as follows:

$$
\nabla \mathbf{u}=\left(\begin{array}{ll}
\frac{d u_{x}}{d x} & \frac{d u_{x}}{d y} \\
\frac{d u_{y}}{d x} & \frac{d u_{y}}{d y}
\end{array}\right) .
$$

In order to fully characterize the deformation of the lattice, we decompose $J$ into its polar form,

$$
J=W P=W V^{\top} D V
$$

where $W$ is the rotation matrix corresponding to the rotation of the full lattice and the matrix $P$ describes the local anisotropy and scaling. $P$ is further decomposed into the rotation matrix $V$ indicating the orientation of the axis of anisotropy (i.e., the axis of largest scaling, with the axis of smallest scaling perpendicular to it) and the diagonal scaling matrix $D=\left(\begin{array}{cc}d_{1} & 0 \\ 0 & d_{2}\end{array}\right)$, where by convention and implementation $d_{1} \geqslant d_{2}$ holds for any position $\mathbf{r}$.

The geometric mean of these directional scaling factors is equal to the square root of the determinant of $D$ and therefore of $J: \sqrt{d_{1} d_{2}}=\sqrt{\operatorname{det}(J)}$. As this corresponds to the local scaling of the wavelength of the moiré lattice, we can use this to quantify the local twist angle:

$$
\lambda(\mathbf{r})=\sqrt{d_{1} d_{2}} \frac{4 \pi}{\sqrt{3}\left|\mathbf{q}_{j}\right|},
$$


where $\left|\mathbf{q}_{j}\right|$ is the length of the chosen reference vectors. This local wavelength is then converted to a local twist angle using the well-known expression

$$
\theta(\mathbf{r})=2 \arcsin \left(\frac{2 \lambda(\mathbf{r})}{a}\right),
$$

where $a=2.46 \AA$ is the lattice constant of graphene and $\theta(\mathbf{r})$ is the local twist angle.

A quantification of the local anisotropy is given by the ratio $\kappa=d_{1} / d_{2}$, and the angle between the anisotropy axis and the $x$ axis is finally calculated from $V: \psi=\arctan \left(\frac{V_{x y}}{V_{x x}}\right)$.

In our practical implementation, the singular value decomposition (SVD) is used to obtain the decomposition in Eq. (A2) for each point in the image, and MATLAB's ATAN2 is used to find the right quadrant of the angles from the signs of $V_{x x}$ and $V_{x y}$.

\section{Determination of the displacement field $u(r)$}

In order to determine $\mathbf{u}(\mathbf{r})$ for a certain image, we perform a lock-in measurement. To clarify, we can represent any (nearly) periodic image as

$$
T_{m}(\mathbf{r})=T_{0} \sum_{j} e^{i \mathbf{q}_{j} \cdot[\mathbf{r}+\mathbf{u}(\mathbf{r})]}=T_{0} \sum_{j} e^{i\left(\mathbf{q}_{j} \cdot \mathbf{r}+\phi_{j}\right)},
$$

where $\phi_{j}=\mathbf{q}_{j} \cdot \mathbf{u}(\mathbf{r})$ is the position-dependent phase of the lattice. The summation runs over the reciprocal lattice vectors $\mathbf{q}_{j}\left(j \in\{1,2,3\}\right.$ for a hexagonal lattice), $T_{0}$ is the constant indicating the amplitude of the modulation, and $\mathbf{u}(\mathbf{r})$ is again the displacement field.

The phase is measured using standard lock-in procedure: The existing image is mixed with a reference image containing a specific plane wave. If we choose the periodicity of this reference wave sufficiently close to that of the lattice in the image itself, we can then low-pass filter the mixed image and end up with a phase map for a specific wave. For clarification,

$$
\cos \left(\mathbf{q}_{j} \cdot \mathbf{r}+\phi_{j}\right) e^{-i q_{j} \cdot \mathbf{r}}=\frac{e^{i \phi_{j}}}{2}\left(1+e^{-2 i\left(\mathbf{q}_{j} \cdot \mathbf{r}+\phi_{j}\right)}\right) \mapsto \frac{1}{2} e^{i \phi_{j}},
$$

where the cosine in the first term denotes the (real-valued) measured image, whereas the complex exponential denotes the reference wave and $\mapsto$ denotes low-pass filtering in order to get rid of the last term between brackets, corresponding to a rotating wave approximation. Alternatively, for a Gaussian low-pass filter, this corresponds to a real-space Gaussian integration window of the lock-in.

By taking the (pointwise) angle of the complex, filtered product image, we end up with the phase map. In particular, this phase map contains information about the displacements of each pixel in the measured image $T_{m}(\mathbf{r})$ with respect to the pristine reference lattice $T_{r}(\mathbf{r})$ along the wave vector $\mathbf{q}_{j}$ used for the lock-in procedure. This procedure is repeated for at least one additional reciprocal lattice vector. The two phase maps are then used to find the displacement field $\mathbf{u}(\mathbf{r})$. From the definition of $\mathbf{u}(\mathbf{r})$ [Eq. (A1)], the following holds: $\mathbf{r}^{\prime}=\mathbf{r}+\mathbf{u}(\mathbf{r})$. Multiplying this equation by the reciprocal lattice vectors, we get a system of equations expressing the projection of the distortion onto the reciprocal lattice vectors:

$$
\mathbf{q}_{j} \cdot \mathbf{r}^{\prime}=\mathbf{q}_{j} \cdot \mathbf{r}+\phi_{j}, \quad j \in 1,2,3
$$

Selecting only $j \in\{1,2\}$, we have, in matrix notation,

$$
Q=\left(\begin{array}{l}
-\mathbf{q}_{1}- \\
-\mathbf{q}_{2}-
\end{array}\right)=\left(\begin{array}{ll}
q_{1 x} & q_{1 y} \\
q_{2 x} & q_{2 y}
\end{array}\right)
$$

such that we can write, for $\phi=\left(\begin{array}{l}\phi_{1} \\ \phi_{2}\end{array}\right)$,

$$
Q \mathbf{r}^{\prime}=Q \mathbf{r}+\phi
$$

Multiplying by $Q^{-1}$, we find $\mathbf{r}^{\prime}=\mathbf{r}+Q^{-1} \phi$, and therefore $\mathbf{u}(\mathbf{r})=Q^{-1} \phi(\mathbf{r})$.

\section{Additional notes on choice of reference vectors}

\section{a. Selecting two reference vectors}

To obtain $\mathbf{u}(\mathbf{r})$ as described above, we only used the phase of the lock-in signal of two reference vectors. For a triangular or hexagonal lattice, a priori three possible choices of which two reference vectors to use are possible from the three linear independent reference vectors as fitted to the fast Fourier transform (FFT) of the image. To select which two vectors to use for the reconstruction of $\mathbf{u}(\mathbf{r})$, we either selected the ones with the largest average lock-in amplitude or inspected the phase-unwrapped images and selected the ones where no remaining phase slips occurred.

\section{b. Using more than two reference vectors}

In principle, information is lost when only selecting the phase of the lock-in signal of two reference vectors to obtain $\mathbf{u}(\mathbf{r})$. Although not used in this paper, in situations with low signal-to-noise ratio, it could be beneficial to use all the information. Equation (A5) also holds for more than two phases and reference vectors. Although $Q$ is not a square matrix in this case, a solution can be obtained for each pixel using linear least-squares minimization of the following equivalent equation:

$$
Q \mathbf{u}(\mathbf{r})=\phi(\mathbf{r}),
$$

where additionally the amplitudes of the lock-in signals can be used as weights to the minimization problem.

\section{c. Isotropy}

Enforcing the reference lattice to be isotropic can be done either in advance, by enforcing isotropic reference wave vectors (as applied in this paper), or, alternatively, after the initial lock-in step, by adding an additional linear phase $\Delta \phi_{j}=$ $\Delta \mathbf{q}_{j} \cdot \mathbf{r}$ to the obtained phase, where $\Delta \mathbf{q}_{j}$ is the difference between the reference wave vector used and the isotropic wave vector.

The advantage of the latter method would be a slightly improved signal-to-noise ratio, as the smoothing window can be centered around the actual average wave vector occurring in the image instead of the ideal, equal-length, $60^{\circ}$ rotated ones.

\section{APPENDIX B: RELATION OF MOIRÉ LATTICE TO RELATIVE DISPLACEMENT}

For a nonhomogeneous bilayer, the system can be fully described by two displacement fields $\mathbf{u}_{\uparrow}(\mathbf{r})$ and $\mathbf{u}_{\downarrow}(\mathbf{r})$ of the top 
and bottom layer, respectively, compared with an undistorted system.

$$
\begin{aligned}
T_{m}(\mathbf{r}) & =T_{m \uparrow}(\mathbf{r}) \oplus T_{m \downarrow}(\mathbf{r}) \\
& =T_{r \uparrow}\left[\mathbf{r}+\mathbf{u}_{\uparrow}(\mathbf{r})\right] \oplus T_{r \downarrow}\left[\mathbf{r}+\mathbf{u}_{\downarrow}(\mathbf{r})\right] \\
& =T_{r \uparrow}\left(\mathbf{r}_{\uparrow}\right) \oplus T_{r \downarrow}\left(\mathbf{r}_{\downarrow}\right),
\end{aligned}
$$

where $T_{r}(\mathbf{r})$ denote the atomic lattices, $\mathbf{r}_{\downarrow}$ and $\mathbf{r}_{\uparrow}$ denote the lattice coordinates of both lattices, and $\oplus$ denotes the (as of now, unspecified) operation of the combination of two lattices into one image.

We can express the deformation of one atomic lattice with respect to the coordinates of the other:

$$
\begin{aligned}
T_{r \downarrow}\left(\mathbf{r}_{\downarrow}\right) & =T_{r \downarrow}\left[\mathbf{r}+\mathbf{u}_{\downarrow}(\mathbf{r})\right]=T_{r_{\downarrow}}\left[\mathbf{f}_{\downarrow}(\mathbf{r})\right] \\
& =T_{r \downarrow}\left\{\mathbf{f}_{\downarrow}\left[\mathbf{f}_{\uparrow}^{-1}\left(\mathbf{r}_{\uparrow}\right)\right]\right\}=T_{r \downarrow}\left\{\mathbf{f}_{\downarrow}\left[\mathbf{r}_{\uparrow}+\mathbf{u}_{\uparrow}^{\prime}\left(\mathbf{r}_{\uparrow}\right)\right]\right\} \\
& =T_{r \downarrow}\left\{\mathbf{r}_{\uparrow}+\mathbf{u}_{\uparrow}^{\prime}\left(\mathbf{r}_{\uparrow}\right)+\mathbf{u}_{\downarrow}\left[\mathbf{r}_{\uparrow}+\mathbf{u}_{\uparrow}^{\prime}\left(\mathbf{r}_{\uparrow}\right)\right]\right\} .
\end{aligned}
$$

Assert $\mathbf{u}_{\downarrow}(\mathbf{r})=J_{\downarrow} \mathbf{r}+\mathbf{v}_{\downarrow}(\mathbf{r})$, i.e., a global rotation and/or scaling plus local variations. Note that here, $J_{\downarrow}$ is a constant $2 \times 2$ matrix corresponding to a mean $\nabla \mathbf{u}$ and therefore corresponding to $J-I$ in terms of the $J$ defined in Appendix A. In this case, we have

$$
T_{r \downarrow}\left(\mathbf{r}_{\downarrow}\right)=T_{r \downarrow}\left\{\left(I+J_{\downarrow}\right)\left[\mathbf{r}_{\uparrow}+\mathbf{u}_{\uparrow}^{\prime}\left(\mathbf{r}_{\uparrow}\right)\right]+\mathbf{v}_{\downarrow}\left[\mathbf{r}_{\uparrow}+\mathbf{u}_{\uparrow}^{\prime}\left(\mathbf{r}_{\uparrow}\right)\right]\right\} .
$$

For two real lattice plane waves $T_{r}\left(\mathbf{r}^{\prime}\right)=\cos \left(\mathbf{q}_{j} \cdot \mathbf{r}^{\prime}\right)$ and taking the pointwise product for the $\oplus$ operator, we have

$$
\begin{aligned}
T_{m}\left(\mathbf{r}_{\uparrow}\right)= & \cos \left(\mathbf{q}_{j} \mathbf{r}_{\uparrow}\right) \cos \left(\mathbf { q } _ { j } \left\{\left(I+J_{\downarrow}\right)\right.\right. \\
& \left.\left.\times\left[\mathbf{r}_{\uparrow}+\mathbf{u}_{\uparrow}^{\prime}\left(\mathbf{r}_{\uparrow}\right)\right]+\mathbf{v}_{\downarrow}\left[\mathbf{r}_{\uparrow}+\mathbf{u}_{\uparrow}^{\prime}\left(\mathbf{r}_{\uparrow}\right)\right]\right\}\right) \\
= & \cos \left(\mathbf{q}_{j} \mathbf{r}_{\uparrow}\right) \cos \left[\mathbf{q}_{j} \mathbf{r}_{\uparrow}+\delta(\mathbf{r})\right] \\
= & \frac{1}{2} \cos \left[2 \mathbf{q}_{j} \mathbf{r}_{\uparrow}+\delta(\mathbf{r})\right]+\frac{1}{2} \cos [-\delta(\mathbf{r})] \\
= & \frac{1}{2} \cos \left[2 \mathbf{q}_{j} \mathbf{r}_{\uparrow}+\delta(\mathbf{r})\right]+\frac{1}{2} \cos [+\delta(\mathbf{r})] .
\end{aligned}
$$

For the modulation $\delta(\mathbf{r})$ the following holds:

$$
\begin{aligned}
\delta(\mathbf{r}) & =\mathbf{q}_{j}\left\{J_{\downarrow}\left[\mathbf{r}_{\uparrow}+\mathbf{u}_{\uparrow}^{\prime}\left(\mathbf{r}_{\uparrow}\right)\right]+\mathbf{u}_{\uparrow}^{\prime}\left(\mathbf{r}_{\uparrow}\right)+\mathbf{v}_{\downarrow}\left[\mathbf{r}_{\uparrow}+\mathbf{u}_{\uparrow}^{\prime}\left(\mathbf{r}_{\uparrow}\right)\right]\right\} \\
& =\mathbf{q}_{j} J_{\downarrow}\left(\mathbf{r}_{\uparrow}+\mathbf{u}_{\uparrow}^{\prime}\left(\mathbf{r}_{\uparrow}\right)+J_{\downarrow}^{-1}\left\{\mathbf{u}_{\uparrow}^{\prime}\left(\mathbf{r}_{\uparrow}\right)+\mathbf{v}_{\downarrow}\left[\mathbf{r}_{\uparrow}+\mathbf{u}_{\uparrow}^{\prime}\left(\mathbf{r}_{\uparrow}\right)\right]\right\}\right) .
\end{aligned}
$$

Substituting $\mathbf{r}_{\uparrow}=\mathbf{r}+\mathbf{u}_{\uparrow}(\mathbf{r})$ and $\mathbf{u}_{\uparrow}^{\prime}\left(\mathbf{r}_{\uparrow}\right)=-\mathbf{u}_{\uparrow}(\mathbf{r})$,

$$
\begin{aligned}
\delta(\mathbf{r}) & =\mathbf{q}_{j} J_{\downarrow}\left[\mathbf{r}-J_{\downarrow}^{-1} \mathbf{u}_{\uparrow}(\mathbf{r})+J_{\downarrow}^{-1} \mathbf{v}_{\downarrow}(\mathbf{r})\right] \\
& =\mathbf{q}_{j} J_{\downarrow}\left[\mathbf{r}+\mathbf{u}_{\text {moiré }}(\mathbf{r})\right],
\end{aligned}
$$

with $\quad \mathbf{u}_{\text {moiré }}(\mathbf{r})=J_{\downarrow}^{-1}\left[\mathbf{v}_{\downarrow}(\mathbf{r})-\mathbf{u}_{\uparrow}(\mathbf{r})\right]=J_{\downarrow}^{-1} \mathbf{u}_{\sim}(\mathbf{r}), \quad$ where $\mathbf{u} \sim(\mathbf{r})$ denotes the relative displacement between the upper layer and the rotated lower layer. Substituting back in $T_{m}$,

$$
\begin{aligned}
T_{m}(\mathbf{r})= & \frac{1}{2} \cos \left(2 \mathbf{q}_{j}\left\{\mathbf{r}+\mathbf{u}_{\uparrow}(\mathbf{r})+\frac{1}{2}\left[J_{\downarrow} \mathbf{r}-\mathbf{u}_{\uparrow}(\mathbf{r})+\mathbf{v}_{\downarrow}(\mathbf{r})\right]\right\}\right) \\
& +\frac{1}{2} \cos \left\{J_{\downarrow}^{\top} \mathbf{q}_{j}\left[\mathbf{r}+\mathbf{u}_{\text {moiré }}(\mathbf{r})\right]\right\}, \\
T_{m}(\mathbf{r})= & \frac{1}{2} \cos \left(2 \mathbf{q}_{j}\left\{\mathbf{r}+\frac{1}{2}\left[J_{\downarrow} \mathbf{r}+\mathbf{u}_{\uparrow}(\mathbf{r})+\mathbf{v}_{\downarrow}(\mathbf{r})\right]\right\}\right) \\
& +\frac{1}{2} \cos \left\{J_{\downarrow}^{\top} \mathbf{q}_{j}\left[\mathbf{r}+\mathbf{u}_{\text {moiré }}(\mathbf{r})\right]\right\} .
\end{aligned}
$$

(a)

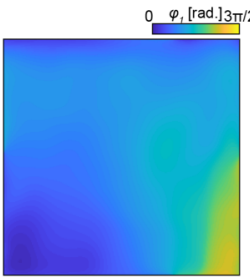

(b)
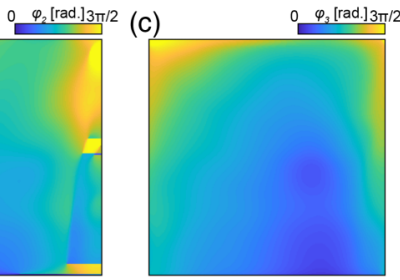

FIG. 5. Phase maps of the data shown in Fig. 4 [and Fig. 8(a)]. (a)-(c) correspond to the phase maps of the Bragg peak labeled $\mathbf{q}_{1}$, $\mathbf{q}_{2}$, and $\mathbf{q}_{3}$, respectively [see Fig. 4(b)]. Because the map corresponding to $\mathbf{q}_{2}$ shows some phase singularities, we use $\phi_{1}$ and $\phi_{2}$ for determining the displacement field.

Note that for a 2D lattice consisting of the sum of two or more cosines, each with its own $\mathbf{q}_{j}$, this construction can be made for each $\mathbf{q}_{j}$ separately, nevertheless resulting in a single, joint $\mathbf{u}_{\text {moiré }}(\mathbf{r})$ (as expected).

For a small twist angle $\theta$ between two equal lattices, e.g., magic angle twisted bilayer graphene, we have

$$
\begin{aligned}
J_{\downarrow} & =R(\theta)-I=\left(\begin{array}{cc}
\cos \theta-1 & -\sin \theta \\
\sin \theta & \cos \theta-1
\end{array}\right) \\
& \approx\left(\begin{array}{cc}
-\frac{1}{2} \theta^{2}+\frac{\theta^{4}}{24} & -\theta+\frac{\theta^{3}}{6} \\
\theta-\frac{\theta^{3}}{6} & -\frac{1}{2} \theta^{2}+\frac{\theta^{4}}{24}
\end{array}\right) \\
& =\theta\left(\begin{array}{cc}
-\frac{1}{2} \theta+\frac{\theta^{3}}{24} & -\left(1-\frac{\theta^{2}}{6}\right) \\
1-\frac{\theta^{2}}{6} & -\frac{1}{2} \theta+\frac{\theta^{3}}{24}
\end{array}\right) \\
& =\theta R\left(\frac{\pi}{2}+\frac{\theta}{2}\right)+\theta^{3}\left(\begin{array}{cc}
\frac{\theta}{48} & -\frac{1}{3} \\
+\frac{1}{3} & \frac{\theta}{48}
\end{array}\right) .
\end{aligned}
$$

Therefore, in this case the topography $T_{m}(\mathbf{r})$ consists of the sum of a cosine with approximately twice the atomic frequency and a cosine with approximately $\theta$ times the atomic frequency: the moiré frequency. As expected, this lattice is rotated by $\frac{\pi}{2}$ plus half the angle of the original rotation, i.e., angled halfway in between both atomic lattices.

\section{Relation to uniaxial strain models}

Graphene has a Poisson ratio $\delta=0.17$, so if a strain $\epsilon$ is applied in one direction, it shrinks in the perpendicular direction by $\delta \epsilon$. By applying the decomposition into $\theta(\mathbf{r}), \kappa(\mathbf{r})$, and $\psi(\mathbf{r})$, as described in Appendix A2, to the relative displacement between the layers $\mathbf{u}_{\sim}(\mathbf{r})$ and assuming that the relative strain is dominated by the strain of one layer, we can calculate that strain $\epsilon(\mathbf{r})$. For uniaxial strain, we have, with these assumptions in terms of the decomposition into relative displacement,

$$
\kappa(\mathbf{r})=\frac{d_{1}}{d_{2}}=\frac{1+\epsilon}{1-\delta \epsilon},
$$

and therefore we can express the strain of a single layer as follows:

$$
\epsilon(\mathbf{r})=\frac{d_{1}-d_{2}}{d_{2}+\delta d_{1}},
$$




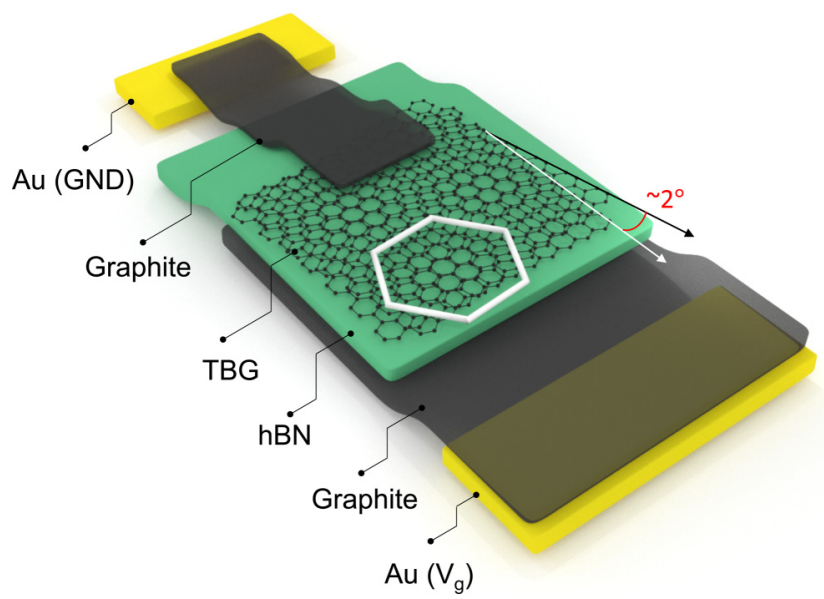

FIG. 6. Schematic overview of the devices studied in this paper. The contact labeled GND can be used to bias or ground the bilayer.

which can then be related to other measurements and models [22,34]. In Appendix J, we discuss the accuracy of these models. Note that the measured quantity $\mathbf{u}_{\text {moire }}(\mathbf{r})$ is related to the relative displacement by a multiplication of $J^{-1}$. For small twist angles, $\left\|J^{-1}\right\| \approx \frac{1}{\theta}$ (with $\theta$ in radians, i.e., for $\theta=1.05^{\circ}$ we have $\left\|J^{-1}\right\| \approx 55$ ), strongly amplifying effects of small relative displacement.

\section{APPENDIX C: PHASE UNWRAPPING AND SINGULARITIES}

In this paper, phase unwrapping of a periodic phase is needed in two separate places: in the unwrapping of the lock- in phase $\phi_{j}(\mathbf{r})$ before reconstructing $\mathbf{u}(\mathbf{r})$, and to obtain a single-valued anisotropy angle $\psi(\mathbf{r})$. The phase is unwrapped in both directions of the image. The order in which this is done usually does not matter, provided there are no phase singularities present in the image. We occasionally encountered some phase singularities in one of the three phase maps (Fig. 5), but we worked around this simply by using the other two phase maps in order to find the displacement field.

In case this is not an option, for example, when applying this technique to a square lattice, and/or when phase slips are present in all phase maps, there are more sophisticated algorithms for phase unwrapping available [31,39,40].

Some of these phase slips were present in the $\psi(\mathbf{r})$ maps, for example, the one displayed in Fig. 4(e). Here, we used a MATLAB implementation of a least-squares-based phase unwrapping algorithm $[39,41]$.

\section{APPENDIX D: DEVICE OVERVIEW}

A schematic of the devices studied in this paper is presented in Fig. 6. More information about the actual fabrication process can be found in the main text.

\section{APPENDIX E: ACCURACY OF THE ALGORITHM}

As an additional consistency check, we used the LawlerFujita algorithm to reconstruct the undistorted image [32] and then applied the algorithm on the undistorted image in order to extract the residual displacement field and compare it to the previously extracted displacement field. Here, a perfectly performing and consistent algorithm would extract a zero residual displacement field. Therefore this gives an indication of the error of the quantities extracted by the algorithm. Since (a)

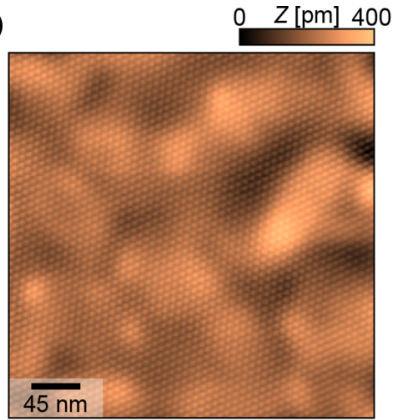

(d)

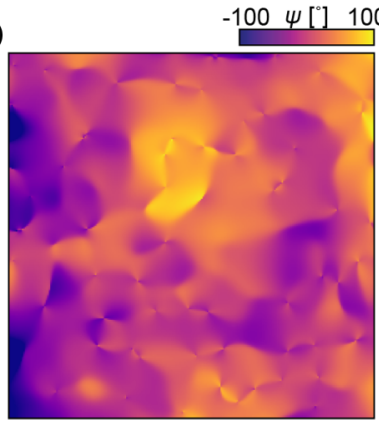

(b)

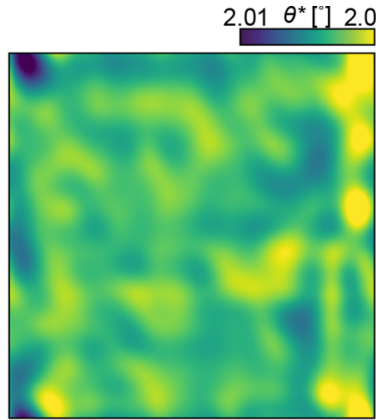

(e)

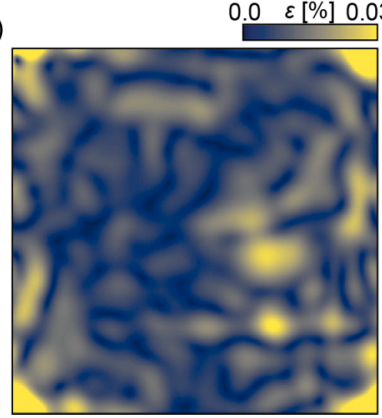

(c)

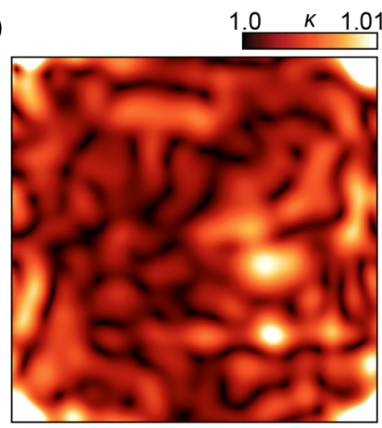

(f)

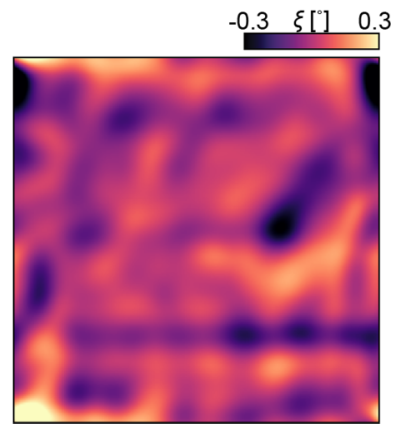

FIG. 7. (a) Lawler-Fujita corrected STM topography of Fig. 4(a) [and Fig. 8(a)]. (b) Extracted effective twist angle map of (a). (c) Extracted residual local anisotropy map of (a). (d) Local anisotropy angle of (c). (e) Heterostrain map of (a). (f) Local moiré rotation of (a). This angle corresponds to the angle in the $W$ matrix [Eq. (A2)]. 
(a)

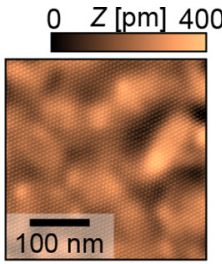

(b)

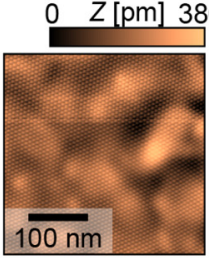

(c)

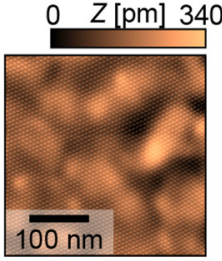

(d)

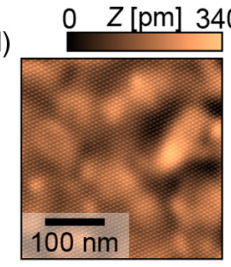

(e)

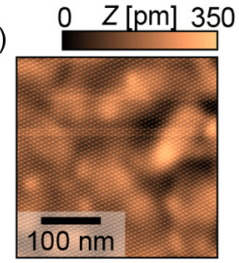

(f)

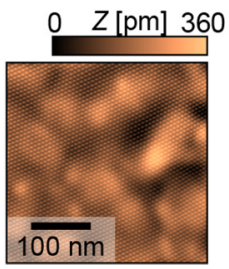

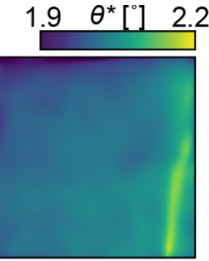

$1.9 \theta^{*}\left[{ }^{\circ}\right] 2.2$

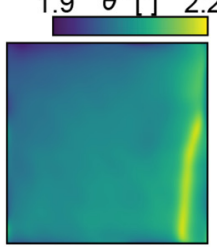

$1.9 \theta^{*}\left[{ }^{\circ}\right]$

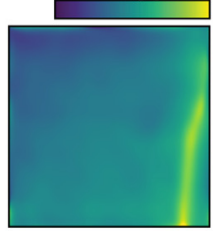

$1.9 \theta^{*}\left[{ }^{\circ}\right] \quad 2$
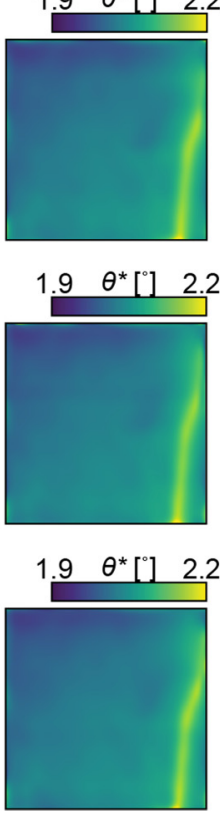
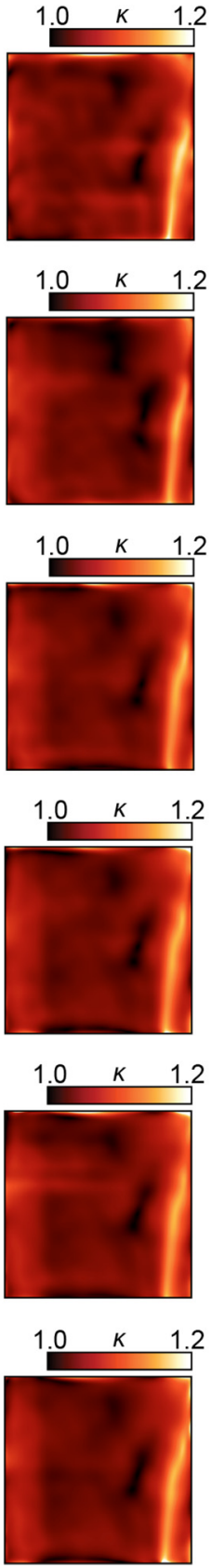
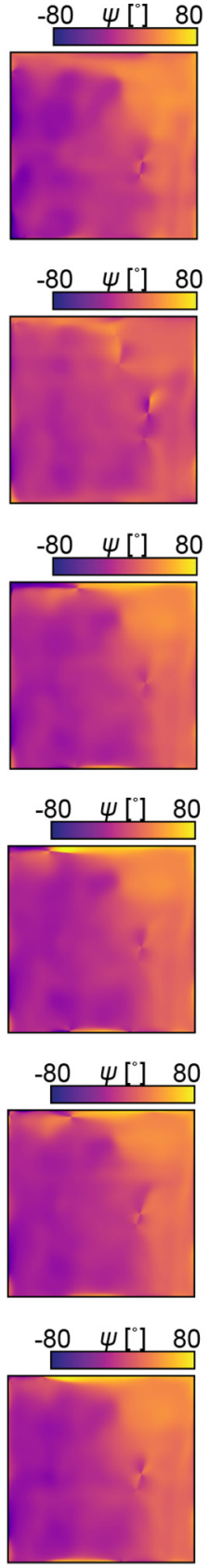
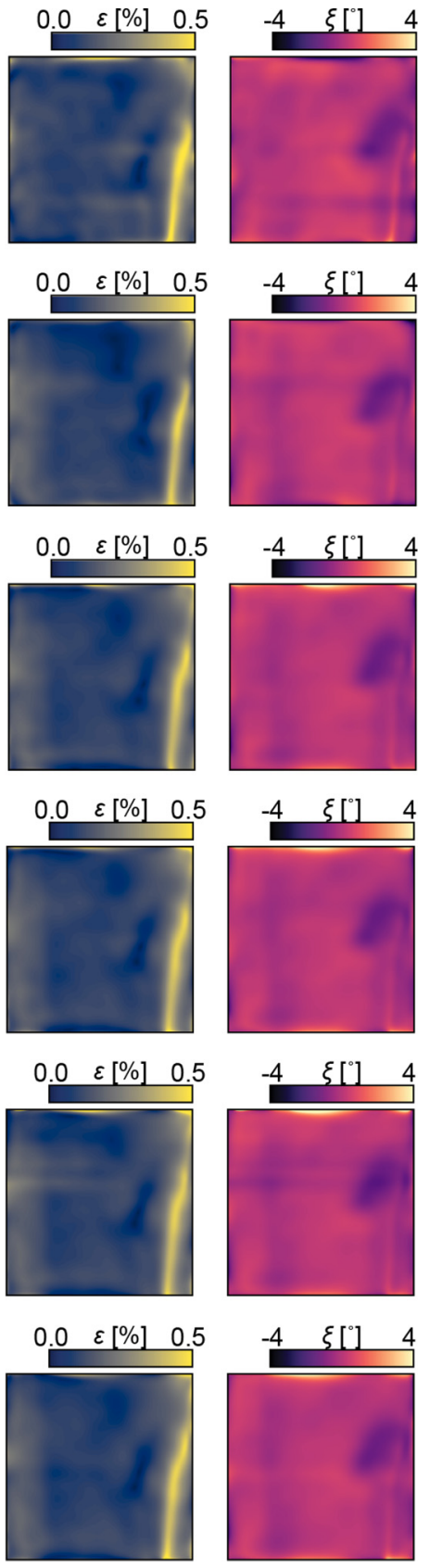

FIG. 8. Spatial lock-in output for sequentially measured topographies in the same field of view. (f) was measured at $65 \mathrm{~nm} / \mathrm{s}$, whereas (a)-(e) were measured with a scan speed of $54 \mathrm{~nm} / \mathrm{s}$. The setup condition was kept constant between measurements: $V=250 \mathrm{mV}$, $I=20 \mathrm{pA}$.

we decompose the displacement field, for an almost zero displacement field, we expect the effective twist angle map to become more centered around the average twist angle (in this case, $2.02^{\circ}$ ). Furthermore, we expected that most of the anisotropy is gone, i.e., $\kappa \rightarrow 1$ and $\epsilon \rightarrow 0$.

We check this using the topography presented in Fig. 4 [and in Fig. 8(a)] and show the results in Fig. 7. Aside from edge effects in the corner, both the residual anisotropy and the residual variations in the twist angle are more than an order of magnitude smaller than the originally obtained values, indicating self-consistency of the algorithm.

\section{APPENDIX F: VALIDITY CHECK WITH MORE DATA}

In this paper, we claim that the contribution of piezo drift to the output of our algorithm is negligible. To verify this, we apply it to multiple topographies, all sequentially measured on the same area. All of them are measured with a scan speed of $54 \mathrm{~nm} / \mathrm{s}$, except the last one [Fig. 8(f)], which is measured at $65 \mathrm{~nm} / \mathrm{s}$. Because piezo drift changes with time and scan speed, comparing these data sets provides us with insight into the degree to which the algorithm output is affected by this effect. 

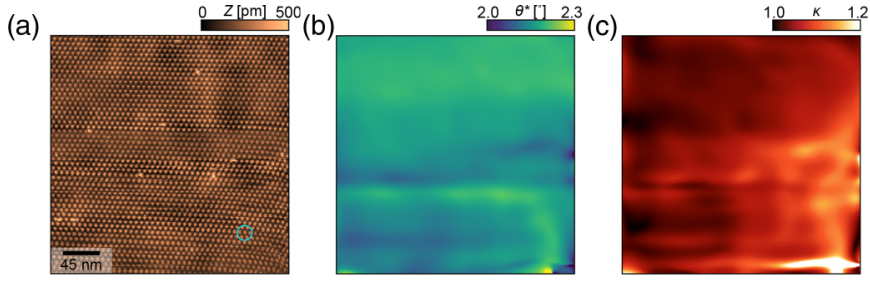

(d)
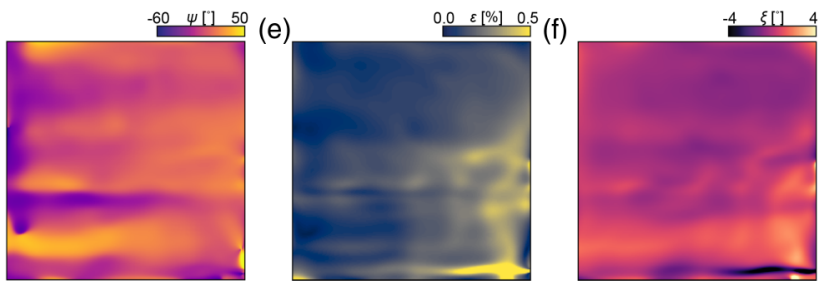

FIG. 9. (a) STM topography of a TBG device with an average twist angle of $2.16^{\circ}$ (setup conditions: $V=170 \mathrm{mV}, I=20 \mathrm{pA}$ ). (b) Extracted effective twist angle map of (a). (c) Extracted local anisotropy map of (a). (d) Local anisotropy angle of (c). (e) Heterostrain map of (a). (f) Local moiré rotation of (a). This angle corresponds to the angle in the $W$ matrix [Eq. (A2)].

The algorithm output for these measurements is displayed in Fig. 8, where Fig. 8(a) corresponds to the data shown in the main text. For completeness, we also show $\xi(\mathbf{r})$, the angle corresponding to the matrix $W$ (see Appendix A 2).

Comparing these results from different scans, we observe that almost all deformations are reproduced, in particular, the vertical linelike feature on the right and the two minima in $\kappa(\mathbf{r})$. The only features not reproduced are horizontal "creases," corresponding to line-to-line scan artifacts. Additionally, no significant difference is observed for Fig. 8(f) with the deviating scan speed compared with the rest. From this, we conclude that most observed deformations are intrinsic to the sample.

\section{APPENDIX G: HETEROGENEITY COMPARISON WITH OTHER DEVICES AND DATA OVERVIEW}

We measured two additional devices, with average twist angles of $2.16^{\circ}$ and $2.01^{\circ}$. The output of the spatial lock-in algorithm for these topographies is displayed in Figs. 9 and 10. Calculating the standard deviation for the twist angle maps, we find $0.03^{\circ}$ and $0.06^{\circ}$, respectively, which is consistent with the result presented in the main text.

In total, topographies from three different devices are presented in this paper. We give a short overview of the data measured per device and the number of pixels for each measurement in Table I.

\section{APPENDIX H: HOMOGENEITY QUANTIFICATION}

In the main text, the average, standard deviation, and peakto-peak spread are given for a cropped area of the twist angle map displayed in Fig. 4(c). We find an average twist angle of $2.02^{\circ}$ with a standard deviation of $0.02^{\circ}$ and a peak-to-peak variation of $0.08^{\circ}$. In Fig. 11, we show the topography, along
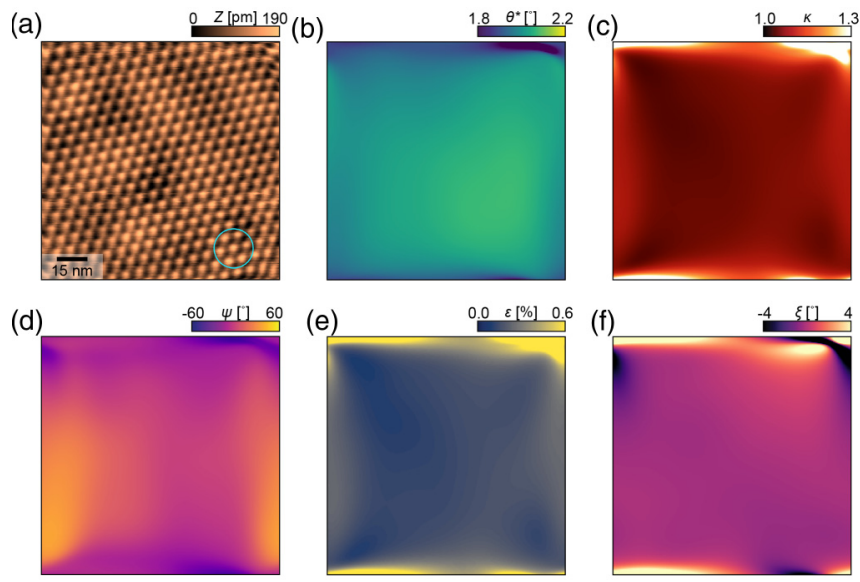

FIG. 10. (a) STM topography of a TBG device with an average twist angle of $2.01^{\circ}$ (setup conditions: $V=350 \mathrm{mV}, I=100 \mathrm{pA}$ ). (b) Extracted effective twist angle map of (a). (c) Extracted local anisotropy map of (a). (d) Local anisotropy angle of (c). (e) Heterostrain map of (a). (f) Local moiré rotation of (a). This angle corresponds to the angle in the $W$ matrix [Eq. (A2)].

with the extracted twist angle map and the crop over which these values are calculated.

Another thing to consider here is some border effects that appear in our data. Because our method is based on lock-in techniques and the real-space resolution is determined by the size of the filter we choose (see main text), we can expect an area around the border of our images to be affected by artifacts. We consider the size of this border to be about two times the radius of the filter used for the spatial lock-in procedure, motivated by the Gaussian profile of the filter: Two times the sigma of a Gaussian covers roughly $95 \%$ of the weight of the window. In Fig. 12, we show the extracted twist angle map, the local anisotropy, and the heterostrain map of Fig. 4(a), accompanied by histograms of each map both including and excluding the border.

\section{APPENDIX I: ARTIFICIAL RESOLUTION LIMITATION FOR COMPARISON WITH SOT}

In order to compare our results with the result for twist angle homogeneity obtained on an encapsulated device from SQUID-on-tip (SOT) measurements [14], we have to consider

TABLE I. Overview of the measured data per device.

\begin{tabular}{lcc}
\hline \hline Device & Fig. & Pixels $(n)$ \\
\hline 1 & $2(\mathrm{a})$ & $321 \times 321$ \\
2 & $2(\mathrm{c}), 4,7,8(\mathrm{a})$, and 11 & $246 \times 246$ \\
2 & $8(\mathrm{~b})-8(\mathrm{f})$ & $984 \times 984$ \\
$2^{\mathrm{a}}$ & 10 & $256 \times 256$ \\
3 & 9 & $236 \times 236$ \\
\hline
\end{tabular}

$\overline{\bar{a} \text { Measured after moving a few micrometers from the initial field of }}$ view. 

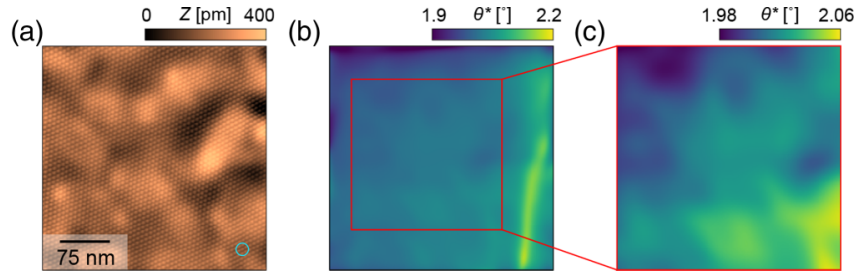

FIG. 11. (a) STM topography of a device with an average twist angle of $2.02^{\circ}[V=250 \mathrm{mV}, I=20 \mathrm{pA}$, same data as in Fig. 4(a)]. (b) Effective twist angle map extracted from (a), by the algorithm discussed. The red square indicates the area over which the average twist angle and standard deviation are calculated. (c) Effective twist angle map corresponding to the area marked by the red square in (b).

the resolution of SOT ( $\sim 30 \mathrm{~nm})$. To this end, we smear our obtained twist angle map [Fig. 4(c)] with a Gaussian filter with a width of $\sigma=15 \mathrm{~nm}$, the result of which is shown in Fig. 13 . Then, we obtain a peak-to-peak value of $0.20^{\circ}$ and a standard deviation of $0.036^{\circ}$.

\section{APPENDIX J: ERROR ESTIMATION OF THE HETEROSTRAIN MODEL}

In previous STM work, the twist angle of twisted bilayer graphene has been extracted with a heterostrain model [22]. This model relies heavily on accurately fitting a Gaussian to the moiré lattice sites in order to extract their position in space and, thereby, the relative distance between neighboring sites.
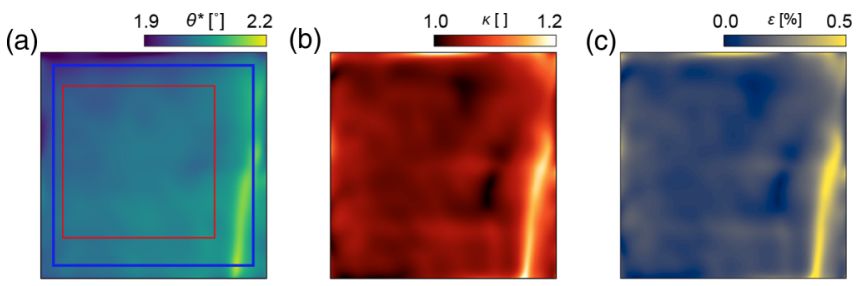

(d)

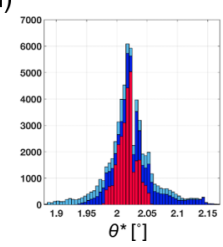

(e)

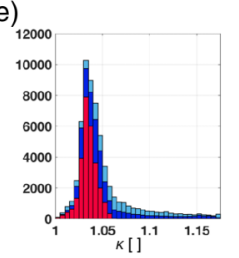

(f)

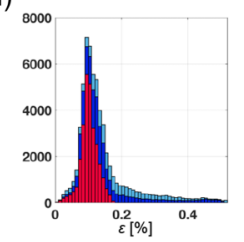

FIG. 12. (a) Effective twist angle map extracted from the data shown in Fig. 4(a). (b) Local moiré anisotropy map $\kappa(\mathbf{r})$ extracted from the data shown in Fig. 4(a). (c) Heterostrain map extracted from the data shown in Fig. 4(a). (d)-(f) Histograms of the maps displayed above. The light blue histograms count the full data as shown, whereas the dark blue histograms exclude a border of two times the filter radius used in the lock-in procedure [pixels outside of the dark blue border in (a)]. Finally, the red histograms count the data inside the area marked by the red square in (a).
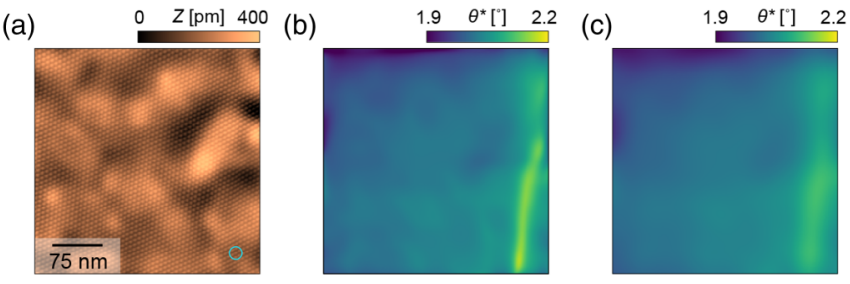

FIG. 13. (a) STM topography of a device with an average twist angle of $2.02^{\circ}[V=250 \mathrm{mV}, I=20 \mathrm{pA}$, same data as in Fig. 4(a) . (b) Effective twist angle map extracted from (a), by the algorithm discussed. (c) Twist angle map of (b) after smearing with a Gaussian filter with a sigma of $15 \mathrm{~nm}$.

Here, a Gaussian is fitted to a representative example of the moiré sites in our data, and we calculate the $95 \%$ confidence interval. The result is shown in Fig. 14. We find a diameter of $\sim 1 \mathrm{~nm}$ for the $95 \%$ confidence interval.

\section{APPENDIX K: TWIST ANGLE HOMOGENEITY OVERVIEW}

In this Appendix, we provide the extracted twist angle homogeneity of each device presented in this paper (Table II).

\section{APPENDIX L: DATA PROCESSING}

Regarding data preprocessing and postprocessing, we made the following manipulations:

(i) Topographies are obtained from the measured data by subtracting a polynomial background up to eighth order. It was verified that this did not significantly influence the extracted displacement fields.

(ii) The topography in Fig. 2(a) was additionally line subtracted.

(iii) FFTs are calculated from the periodic part of the data, after applying the periodic + smooth decomposition algorithm [42].

(iv) The FFT in Fig. 4(b) uses interpolative shading.

(v) The FFT in Fig. 2(b) is furthermore smeared with a Gaussian filter (with a width of $\sigma=0.5$ pixels). (a)

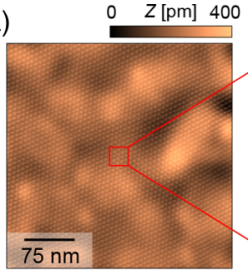

(b)

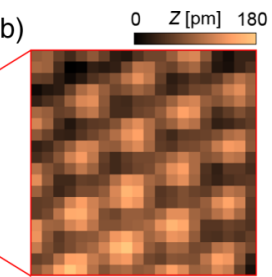

(c)

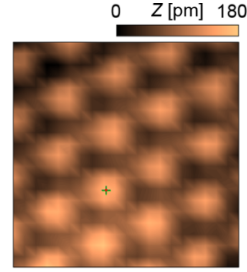

FIG. 14. (a) STM topography of a device with an average twist angle of $2.02^{\circ}[\mathrm{V}=250 \mathrm{mV}, I=20 \mathrm{pA}$, same data as in Fig. 4(a) $]$. (b) Zoom-in of (a). (c) Same as in (b), but plotted with interpolative shading. The red marker indicates the position of the Gaussian fitted to that moiré site, and the green cross represents the extent of the $95 \%$ confidence interval. 
TABLE II. Overview of the twist angle homogeneity extracted per device. Blue and red areas refer to the data inside the areas marked by the blue and red squares in Fig. 12, respectively.

\begin{tabular}{lccc}
\hline \hline Description & Average twist angle & Peak-to-peak variation & Standard deviation \\
\hline Full field of view, Fig. 12 & $2.02^{\circ}$ & $0.29^{\circ}$ & $0.039^{\circ}$ \\
Blue area, Fig. 12 & $2.02^{\circ}$ & $0.23^{\circ}$ & $0.033^{\circ}$ \\
Red area, Fig. 12 & $2.02^{\circ}$ & $0.08^{\circ}$ & $0.015^{\circ}$ \\
Full field of view, Fig. 12, after correcting PSF & $2.02^{\circ}$ & $0.20^{\circ}$ & $0.036^{\circ}$ \\
Full field of view, Fig. 9 (blue area equivalent) & $2.16^{\circ}$ & $0.14^{\circ}$ & $0.029^{\circ}$ \\
Full field of view, Fig. 10 (blue area equivalent) & $2.03^{\circ}$ & & $0.029^{\circ}$ \\
\hline \hline
\end{tabular}

[1] R. Bistritzer and A. H. MacDonald, Moiré bands in twisted double-layer graphene, Proc. Natl. Acad. Sci. USA 108, 12233 (2011).

[2] M. P. Allan, M. H. Fischer, O. Ostojic, and A. Andringa, Creating better superconductors by periodic nanopatterning, SciPost Phys. 3, 010 (2017).

[3] Y. Cao, V. Fatemi, A. Demir, S. Fang, S. L. Tomarken, J. Y. Luo, J. D. Sanchez-Yamagishi, K. Watanabe, T. Taniguchi, E. Kaxiras, R. C. Ashoori, and P. Jarillo-Herrero, Correlated insulator behaviour at half-filling in magic-angle graphene superlattices, Nature (London) 556, 80 (2018).

[4] Y. Cao, V. Fatemi, S. Fang, K. Watanabe, T. Taniguchi, E. Kaxiras, and P. Jarillo-Herrero, Unconventional superconductivity in magic-angle graphene superlattices, Nature (London) 556, 43 (2018).

[5] X. Lu, P. Stepanov, W. Yang, M. Xie, M. A. Aamir, I. Das, C. Urgell, K. Watanabe, T. Taniguchi, G. Zhang, A. Bachtold, A. H. MacDonald, and D. K. Efetov, Superconductors, orbital magnets and correlated states in magic-angle bilayer graphene, Nature (London) 574, 653 (2019).

[6] H. Polshyn, M. Yankowitz, S. Chen, Y. Zhang, K. Watanabe, T. Taniguchi, C. R. Dean, and A. F. Young, Large linear-intemperature resistivity in twisted bilayer graphene, Nat. Phys. 15, 1011 (2019).

[7] A. L. Sharpe, E. J. Fox, A. W. Barnard, J. Finney, K. Watanabe, T. Taniguchi, M. A. Kastner, and D. GoldhaberGordon, Emergent ferromagnetism near three-quarters filling in twisted bilayer graphene, Science 365, 605 (2019).

[8] M. Yankowitz, S. Chen, H. Polshyn, Y. Zhang, K. Watanabe, T. Taniguchi, D. Graf, A. F. Young, and C. R. Dean, Tuning superconductivity in twisted bilayer graphene, Science $\mathbf{3 6 3}$, 1059 (2019).

[9] P. Stepanov, I. Das, X. Lu, A. Fahimniya, K. Watanabe, T. Taniguchi, F. H. L. Koppens, J. Lischner, L. Levitov, and D. K. Efetov, Untying the insulating and superconducting orders in magic-angle graphene, Nature (London) 583, 375 (2020).

[10] E. Codecido, Q. Wang, R. Koester, S. Che, H. Tian, R. Lv, S. Tran, K. Watanabe, T. Taniguchi, F. Zhang, M. Bockrath, and C. N. Lau, Correlated insulating and superconducting states in twisted bilayer graphene below the magic angle, Sci. Adv. 5, eaaw9770 (2019).

[11] Y. Saito, J. Ge, K. Watanabe, T. Taniguchi, and A. F. Young, Independent superconductors and correlated insulators in twisted bilayer graphene, Nat. Phys. 16, 926 (2020).
[12] Y. Cao, D. Chowdhury, D. Rodan-Legrain, O. RubiesBigorda, K. Watanabe, T. Taniguchi, T. Senthil, and P. Jarillo-Herrero, Strange Metal in Magic-Angle Graphene with Near Planckian Dissipation, Phys. Rev. Lett. 124, 076801 (2020).

[13] L. Balents, C. R. Dean, D. K. Efetov, and A. F. Young, Superconductivity and strong correlations in moiré flat bands, Nat. Phys. 16, 725 (2020).

[14] A. Uri, S. Grover, Y. Cao, J. A. Crosse, K. Bagani, D. Rodan-Legrain, Y. Myasoedov, K. Watanabe, T. Taniguchi, P. Moon, M. Koshino, P. Jarillo-Herrero, and E. Zeldov, Mapping the twist-angle disorder and Landau levels in magic-angle graphene, Nature (London) 581, 47 (2020).

[15] S. Lisi, X. Lu, T. Benschop, T. A. de Jong, P. Stepanov, J. R. Duran, F. Margot, I. Cucchi, E. Cappelli, A. Hunter, A. Tamai, V. Kandyba, A. Giampietri, A. Barinov, J. Jobst, V. Stalman, M. Leeuwenhoek, K. Watanabe, T. Taniguchi, L. Rademaker et al., Observation of flat bands in twisted bilayer graphene, Nat. Phys. (2020), doi: 10.1038/s41567-020-01041-x.

[16] I. Razado-Colambo, J. Avila, J. P. Nys, C. Chen, X. Wallart, M. C. Asensio, and D. Vignaud, NanoARPES of twisted bilayer graphene on SiC: absence of velocity renormalization for small angles, Sci. Rep. 6, 27261 (2016).

[17] A. J. H. Jones, R. Muzzio, P. Majchrzak, S. Pakdel, D. Curcio, K. Volckaert, D. Biswas, J. Gobbo, S. Singh, J. T. Robinson, K. Watanabe, T. Taniguchi, T. K. Kim, C. Cacho, N. Lanata, J. A. Miwa, P. Hofmann, J. Katoch, and S. Ulstrup, Observation of electrically tunable van Hove singularities in twisted bilayer graphene from nanoARPES, Adv. Mater. (Weinheim) 32, 2001656 (2020).

[18] L. J. McGilly, A. Kerelsky, N. R. Finney, K. Shapovalov, E.-M. Shih, A. Ghiotto, Y. Zeng, S. L. Moore, W. Wu, Y. Bai, K. Watanabe, T. Taniguchi, M. Stengel, L. Zhou, J. Hone, X. Zhu, D. N. Basov, C. Dean, C. E. Dreyer, and A. N. Pasupathy, Visualization of moiré superlattices, Nat. Nanotechnol. 15, 580 (2020).

[19] S. S. Sunku, A. S. McLeod, T. Stauber, H. Yoo, D. Halbertal, G. Ni, A. Sternbach, B.-Y. Jiang, T. Taniguchi, K. Watanabe, P. Kim, M. M. Fogler, and D. N. Basov, Nano-photocurrent mapping of local electronic structure in twisted bilayer graphene, Nano Lett. 20, 2958 (2020).

[20] U. Zondiner, A. Rozen, D. Rodan-Legrain, Y. Cao, R. Queiroz, T. Taniguchi, K. Watanabe, Y. Oreg, F. von Oppen, A. Stern, E. Berg, P. Jarillo-Herrero, and S. Ilani, Cascade of phase 
transitions and Dirac revivals in magic-angle graphene, Nature (London) 582, 203 (2020).

[21] Y. Choi, J. Kemmer, Y. Peng, A. Thomson, H. Arora, R. Polski, Y. Zhang, H. Ren, J. Alicea, G. Refael, F. von Oppen, K. Watanabe, T. Taniguchi, and S. Nadj-Perge, Electronic correlations in twisted bilayer graphene near the magic angle, Nat. Phys. 15, 1174 (2019).

[22] A. Kerelsky, L. J. McGilly, D. M. Kennes, L. Xian, M. Yankowitz, S. Chen, K. Watanabe, T. Taniguchi, J. Hone, C. Dean, A. Rubio, and A. N. Pasupathy, Maximized electron interactions at the magic angle in twisted bilayer graphene, Nature (London) 572, 95 (2019).

[23] Y. Xie, B. Lian, B. Jäck, X. Liu, C.-L. Chiu, K. Watanabe, T. Taniguchi, B. A. Bernevig, and A. Yazdani, Spectroscopic signatures of many-body correlations in magicangle twisted bilayer graphene, Nature (London) 572, 101 (2019).

[24] D. Wong, K. P. Nuckolls, M. Oh, B. Lian, Y. Xie, S. Jeon, K. Watanabe, T. Taniguchi, B. A. Bernevig, and A. Yazdani, Cascade of electronic transitions in magic-angle twisted bilayer graphene, Nature (London) 582, 198 (2020).

[25] Y. Jiang, X. Lai, K. Watanabe, T. Taniguchi, K. Haule, J. Mao, and E. Y. Andrei, Charge order and broken rotational symmetry in magic-angle twisted bilayer graphene, Nature (London) 573, 91 (2019).

[26] K. P. Nuckolls, M. Oh, D. Wong, B. Lian, K. Watanabe, T. Taniguchi, B. A. Bernevig, and A. Yazdani, Strongly correlated Chern insulators in magic-angle twisted bilayer graphene, Nature (London) 588, 610 (2020).

[27] G. Li, A. Luican, and E. Y. Andrei, Self-navigation of a scanning tunneling microscope tip toward a micronsized graphene sample, Rev. Sci. Instrum. 82, 073701 (2011).

[28] M. Hÿtch, Geometric phase analysis of high resolution electron microscope images, Scanning Microsc. 11, 53 (1997).

[29] Y. Zhu, C. Ophus, J. Ciston, and H. Wang, Interface lattice displacement measurement to $1 \mathrm{pm}$ by geometric phase analysis on aberration-corrected HAADF STEM images, Acta Mater. 61, 5646 (2013).

[30] H. Zhang, Z. Liu, H. Wen, H. Xie, and C. Liu, Subset geometric phase analysis method for deformation evaluation of HRTEM images, Ultramicroscopy 171, 34 (2016).
[31] Q. Kemao, Two-dimensional windowed Fourier transform for fringe pattern analysis: Principles, applications and implementations, Opt. Lasers Eng. 45, 304 (2007).

[32] M. J. Lawler, K. Fujita, J. Lee, A. R. Schmidt, Y. Kohsaka, C. K. Kim, H. Eisaki, S. Uchida, J. C. Davis, J. P. Sethna, and E.-A. Kim, Intra-unit-cell electronic nematicity of the high- $T_{\mathrm{c}}$ copperoxide pseudogap states, Nature (London) 466, 347 (2010).

[33] J. A. Slezak, J. Lee, M. Wang, K. McElroy, K. Fujita, B. M. Andersen, P. J. Hirschfeld, H. Eisaki, S. Uchida, and J. C. Davis, Imaging the impact on cuprate superconductivity of varying the interatomic distances within individual crystal unit cells, Proc. Natl. Acad. Sci. USA 105, 3203 (2008).

[34] Z. Bi, N. F. Q. Yuan, and L. Fu, Designing flat bands by strain, Phys. Rev. B 100, 035448 (2019).

[35] G. Li, A. Luican, J. M. B. Lopes dos Santos, A. H. Castro Neto, A. Reina, J. Kong, and E. Y. Andrei, Observation of van Hove singularities in twisted graphene layers, Nat. Phys. 6, 109 (2010).

[36] C. Dutreix, H. González-Herrero, I. Brihuega, M. I. Katsnelson, C. Chapelier, and V. T. Renard, Measuring the Berry phase of graphene from wavefront dislocations in Friedel oscillations, Nature (London) 574, 219 (2019).

[37] N. N. T. Nam and M. Koshino, Lattice relaxation and energy band modulation in twisted bilayer graphene, Phys. Rev. B 96, 075311 (2017).

[38] L. Balents, General continuum model for twisted bilayer graphene and arbitrary smooth deformations, SciPost Phys. 7, 48 (2019).

[39] D. C. Ghiglia and L. A. Romero, Robust two-dimensional weighted and unweighted phase unwrapping that uses fast transforms and iterative methods, J. Opt. Soc. Am. A 11, 107 (1994).

[40] M. A. Herráez, D. R. Burton, M. J. Lalor, and M. A. Gdeisat, Fast two-dimensional phase-unwrapping algorithm based on sorting by reliability following a noncontinuous path, Appl. Opt. 41, 7437 (2002).

[41] M. F. Kasim, 2D weighted phase unwrapping, MATLAB central file exchange, 2016, https://www.mathworks.com/ matlabcentral/fileexchange/60345-2d-weighted-phaseunwrapping.

[42] L. Moisan, Periodic plus smooth image decomposition, J. Math. Imaging Vis. 39, 161 (2010). 